\title{
BIFURCATIONS IN TWO-DIMENSIONAL REVERSIBLE MAPS
}

\section{T. POST and H.W. CAPEL}

Instituut voor Theoretische Fysica, Universiteit van Amsterdam, Valckenierstraat 65 , 1018 XE Amsterdam, The Netherlands

\section{G.R.W. QUISPEL ${ }^{1}$}

Department of Theoretical Physics, Research School of Physical Sciences, The Australian National University, Canberra, ACT 2601, Australia and Mathematics Research Section, School of Mathematical Sciences, The Australian National University, Canberra, ACT 2601, Australia

\section{J.P. VAN DER WEELE}

Centrum voor Theoretische Natuurkunde, Universiteit Twente, Postbus 217, 7500 AE Enschede, The Netherlands

Received 15 November 1989

We give a treatment of the non-resonant bifurcations involving asymmetric fixed points with Jacobian $\mathscr{I} \neq 1$ in reversible mappings of the plane. These bifurcations include the saddlenode bifurcation not in the neighbourhood of a fixed point with $\mathscr{F}=1$, as well as the so-called transcritical bifurcations and generalized Rimmer bifurcations taking place at a fixed point with Jacobian $\mathscr{F}=1$. The bifurcations are illustrated by some simple examples of model maps. The Rimmer type of bifurcation, with e.g. a center point with $\mathscr{J}=1$ changing into a saddle with Jacobian $\mathscr{J}=1$, an attractor and a repeller, occurs under more general conditions, i.e. also in non-reversible mappings if only a certain order of local reversibility is satisfied. These Rimmer bifurcations are important in connection with the emergence of dissipative features in non-measure-preserving reversible dynamical systems.

\section{Introduction}

Reversible maps can be an adequate tool for the description of physical systems that display conservative behaviour in certain parts of their phase space, and dissipative behaviour in other parts. These two kinds of behaviour, coexisting in the same physical system, have been discussed in refs. [1-7].

\footnotetext{
${ }^{1}$ Present address: Department of Mathematics, La Trobe University, Bundoora, Victoria 3083, Australia.
} 
A map $T$ is called reversible if there exists a decomposition

$$
T=I_{1} I_{2},
$$

where $I_{1}$ and $I_{2}$ are involutions, i.e. maps which are their own inverse,

$$
I_{1}^{2}=I_{2}^{2}=1
$$

The involutions $I_{1}, I_{2}$ are often called symmetries of the map $T$, because for each invariant set $\mathrm{M}$ of the map $T: T \circ \mathrm{M}=\mathrm{M}$, we can easily show that the "reflections" $I_{1} \circ \mathrm{M}$ and $I_{2} \circ \mathrm{M}$ are also invariant under $T$.

(From $T \mathrm{M}=I_{1} I_{2} \mathrm{M}=\mathrm{M}$ we have $I_{2} \mathrm{M}=I_{1} \mathrm{M}$ and hence $T I_{1} \mathrm{M}=I_{1} I_{2} I_{1} \mathrm{M}=$ $I_{1} I_{2} I_{2} \mathrm{M}=I_{1} \mathrm{M}$ and $\left.T I_{2} \mathrm{M}=I_{1} I_{2} I_{2} \mathrm{M}=I_{1} \mathrm{M}=I_{2} \mathrm{M} . \square\right)$

As a typical example we study the two-parameter map (cf. ref. [8])

$$
T:\left\{\begin{array}{c}
x^{\prime}=x+\omega(y) \quad(\bmod 1), \\
y+\frac{K}{2 \pi} g\left(x^{\prime}\right) \\
y^{\prime}=\frac{y-\epsilon y\left(x^{\prime}\right)}{1-}
\end{array}\right.
$$

with $g(x+1)=g(x)$ and $h(x+1)=h(x)$. With the choices $\omega(y)=y, g(x)=$ $\sin (2 \pi x)$, we recover for $\epsilon=0$ the Chirikov-Taylor standard map [9]. If $\omega(-y)=-\omega(y)$ the mapping is reversible with the involutions

$$
\begin{aligned}
& I_{1}:\left\{\begin{array}{l}
x^{\prime}=x \quad(\bmod 1), \\
y^{\prime}=\frac{-y+\frac{K}{2 \pi} g(x)}{1+\epsilon y h(x)},
\end{array}\right. \\
& I_{2}:\left\{\begin{array}{l}
x^{\prime}=x+\omega(y), \\
y^{\prime}=-y .
\end{array}\right.
\end{aligned}
$$

The symmetry curve associated with $I_{1}$ is

$$
[\epsilon h(x)] y^{2}+2 y-\frac{K}{2 \pi} g(x)=0,
$$

while the symmetry curve associated with $I_{2}$ is simply

$$
y=0
$$

The symmetric fixed points of the mapping are given by $y_{0}=0$ and $g\left(x_{0}\right)=0$. If $g(x)$ and $h(x)$ are both odd the map is reversible with the decomposition 
$T=I_{1} I_{2}$ given by

$$
\begin{aligned}
& I_{1}:\left\{\begin{array}{l}
x^{\prime}=-x \quad(\bmod 1), \\
y^{\prime}=\frac{y-\frac{K}{2 \pi} g(x)}{1+\epsilon y h(x)},
\end{array}\right. \\
& I_{2}:\left\{\begin{array}{l}
x^{\prime}=-x-\omega(y), \\
y^{\prime}=y
\end{array}\right.
\end{aligned}
$$

In this case we have the symmetry curves $2 x \in \mathrm{Z}$ of $I_{1}$ and $2 x+\omega(y) \in \mathrm{Z}$ of $I_{2}$. The symmetric fixed points are thus given by $2 x_{0} \in \mathrm{Z}$ and $\omega\left(y_{0}\right) \in \mathrm{Z}$.

The fixed points of the map (1.3) with $g\left(x_{0}\right)=0$ and $y_{0}=0$ trivially have Jacobian $\mathscr{J}=1$. The other fixed points can be divided into two types labeled FP1 and FP2, as follows:

FP1:

$$
K g\left(x_{0}\right)=\epsilon h\left(x_{0}\right)=0 \quad \text { and } \quad \omega\left(y_{0}\right) \in Z ;
$$

FP2:

$$
K g\left(x_{0}\right) \neq 0 \text { and } \epsilon h\left(x_{0}\right) \neq 0 \text { and }-\frac{K}{2 \pi \epsilon} y_{0}^{-2}=\frac{h\left(x_{0}\right)}{g\left(x_{0}\right)}
$$

and $\omega\left(y_{0}\right) \in Z$.

Here the FP2 are always asymmetric fixed points with $\mathscr{g} \neq 1$. The FP1 have Jacobian $\mathscr{g}=1$. They are symmetric fixed points in the case that both $g(x)$ and $h(x)$ are odd, they are asymmetric fixed points when $\omega(y)$ is odd, and $g(x)$ and $h(x)$ are not both odd.

In section 2 we give a stability analysis of the FP1 and FP2. In section 3 we give a description of the non-resonant bifurcations of the map (1.3), i.e. the bifurcations at which FP2 type fixed points can be born, either by a saddlenode bifurcation [10], not in the neighbourhood of one of the FP1, or by a transcritical bifurcation [10] or a Rimmer bifurcation [11-13], taking place at one of the FP1's. Each bifurcation type is illustrated using appropriate choices for $\omega(y), g(x)$ and $h(x)$. Finally, in section 4, we discuss the concept of local reversibility, introduced in ref. [14], and the relations with the various bifurcation scenarios.

\section{Stability analysis}

For the elements of the Jacobian matrix of the map (1.3) we find 


$$
\begin{array}{rlrl}
\frac{\partial x^{\prime}}{\partial x} & =1, & \frac{\partial x^{\prime}}{\partial y} & =\omega^{\prime}(y), \\
\frac{\partial y^{\prime}}{\partial x}=\frac{\frac{K}{2 \pi} g^{\prime}\left(x^{\prime}\right)+\epsilon y y^{\prime} h^{\prime}\left(x^{\prime}\right)}{1-\epsilon y h\left(x^{\prime}\right)}, & \frac{\partial y^{\prime}}{\partial y}=\mp+\omega^{\prime}(y) \frac{\partial y^{\prime}}{\partial x},
\end{array}
$$

where the Jacobian $\mathscr{F}$ is given by

$$
\mathscr{F}=\frac{1+\epsilon y^{\prime} h\left(x^{\prime}\right)}{1-\epsilon y h\left(x^{\prime}\right)}=\frac{y^{\prime}}{y}\left(\frac{y^{\prime}-\frac{K}{2 \pi} g\left(x^{\prime}\right)}{y+\frac{K}{2 \pi} g\left(x^{\prime}\right)}\right) .
$$

For a fixed point $\left(x_{0}, y_{0}\right)$ we have

$$
\begin{gathered}
\left(\frac{\partial y^{\prime}}{\partial x}\right)_{01}=\frac{\frac{K}{2 \pi} g^{\prime}\left(x_{0}\right)+\epsilon y_{01}^{2} h^{\prime}\left(x_{01}\right)}{1-\epsilon y_{0} h\left(x_{01}\right)}, \\
\mathscr{I}_{0}=\frac{1+\epsilon y_{0} h\left(x_{0}\right)}{1-\epsilon y_{01} h\left(x_{0}\right)}=\frac{y_{0}-\frac{K}{2 \pi} g\left(x_{0}\right)}{y_{01}+\frac{K}{2 \pi} g\left(x_{11}\right)}, \\
\operatorname{Tr}_{0}=1+\mathscr{f}_{0}+\omega^{\prime}\left(y_{0}\right)\left(\frac{\partial y^{\prime}}{\partial x}\right)_{11},
\end{gathered}
$$

where $\operatorname{Tr}_{0}$ is the trace of the Jacobian matrix in $\left(x_{0}, y_{0}\right)$. The stability of the fixed points is governed by the eigenvalue equation

$$
\lambda^{2}+\lambda \operatorname{Tr}_{0}+\mathscr{F}_{0}=0
$$

in particular, we have a saddle if $\left|\operatorname{Tr}_{0}\right|>1+\mathscr{f}_{11}$.

For the FP1 we have $\mathscr{f}_{10}=1$ and hence $\left(x_{11}, y_{10}\right)$ is a saddle if

$$
\omega^{\prime}\left(y_{0}\right)\left(\frac{\partial y^{\prime}}{\partial x}\right)_{0}>0 \text { or } \omega^{\prime}\left(y_{0}\right)\left(\frac{\partial y^{\prime}}{\partial x}\right)_{0}<-4 \text {. }
$$

and $\left(x_{0}, y_{0}\right)$ is a center if

$$
-4<\omega^{\prime}\left(y_{01}\right)\left(\frac{\partial y^{\prime}}{\partial x}\right)_{0}<0 .
$$

For the FP2 we have $\mathscr{I}_{0} \neq 1$ and $\left(x_{0}, y_{11}\right)$ is a saddle if 


$$
\omega^{\prime}\left(y_{0}\right)\left(\frac{\partial y^{\prime}}{\partial x}\right)_{0}>0 \text { or } \quad \omega^{\prime}\left(y_{0}\right)\left(\frac{\partial y^{\prime}}{\partial x}\right)_{0}<-2\left(1-\mathscr{J}_{0}\right) \text {. }
$$

Otherwise $\left(x_{0}, y_{0}\right)$ is an attractor $\left(\mathscr{J}_{0}<1\right)$ or a repeller $\left(\mathscr{Z}_{0}>1\right)$.

For future use we observe that $\left(\partial y^{\prime} / \partial x\right)_{0}$ in the case of a FP2 can be written as (cf. eq. (2.3))

$$
\left(\frac{\partial y^{\prime}}{\partial x}\right)_{0}=\frac{\epsilon y_{0}^{2} g^{\prime}\left(x_{0}\right)}{1-\epsilon y_{0} h\left(x_{0}\right)}\left(\frac{h^{\prime}\left(x_{0}\right)}{g^{\prime}\left(x_{0}\right)}-\frac{h\left(x_{0}\right)}{g\left(x_{0}\right)}\right) \text {. }
$$

\section{Bifurcation types}

We shall now analyze the bifurcation scenarios of the FP2. From the definition of the FP2 type fixed points we observe that for reasonable $\omega(y)$ the fixed point condition $\omega\left(y_{0}\right) \in Z$ can only be satisfied for a discrete set of values $y_{0}$. This leads us to consider the condition

$$
\frac{h\left(x_{0}\right)}{g\left(x_{0}\right)}=-\frac{K}{2 \pi \epsilon} y_{0}^{-2}
$$

for fixed values of $y_{0}$.

For our purposes it is now convenient to consider a plot of $z=h(x) / g(x)$ in which also the horizontal line $z=-(K / 2 \pi \epsilon) y_{0}^{-2}$ is drawn. The position of the latter line obviously depends on the map parameters $K$ and $\epsilon$. At the intersections with $z=h(x) / g(x)$ the fixed point condition (3.1) is satisfied. The bifurcation scenarios which emerge are described below.

\subsection{Saddle-node bifurcation}

At a maximum of $h(x) / g(x)$ at $x=x_{\max }$ (where we have no FP1) we have a situation as depicted in fig. 1. Clearly three situations can be distinguished:

(a) $-\frac{K}{2 \pi \epsilon} y_{0}^{-2}>\frac{h\left(x_{\max }\right)}{g\left(x_{\max }\right)}$.

There are no FP2 in the neighbourhood of $x_{\max }$.

(b) $-\frac{K}{2 \pi \epsilon} y_{0}^{-2}=\frac{h\left(x_{\max }\right)}{g\left(x_{\max }\right)}$.

There is one FP2 at $x=x_{0}=x_{\max }$. 


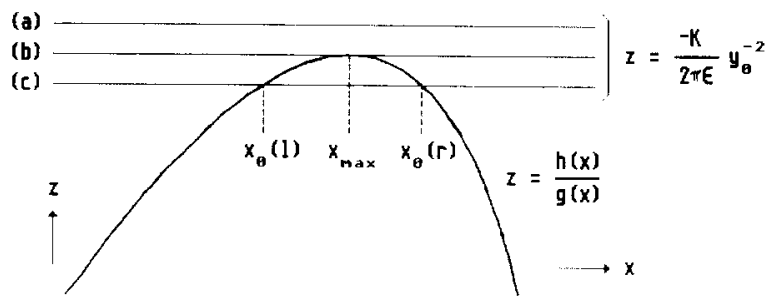

Fig. 1. The function $z=h(x) / g(x)$ near $x_{\max }$. Also drawn are lines $z=-(K / 2 \pi \epsilon) y_{0}{ }^{2}$, for the three situations (a), (b) and (c) discussed in the text. The two fixed points of type FP2 in situation (c) are denoted by $x_{11}(1)$ and $x_{11}(\mathrm{r})$.

(c) $-\frac{K}{2 \pi \epsilon} y_{0}^{-2}<\frac{h\left(x_{\max }\right)}{g\left(x_{\max }\right)}$.

There are two FP2, one with $x_{0}<x_{\max }$ and onc with $x_{11}>x_{\max }$.

We investigate their stability by considering

$$
\omega^{\prime}\left(y_{0}\right)\left(\frac{\partial y^{\prime}}{\partial x}\right)_{0}=\omega^{\prime}\left(y_{01}\right) \frac{K}{2 \pi}\left(\frac{\left[g\left(x_{0}\right)\right]^{2} / h\left(x_{0}\right)}{1+\frac{K}{2 \pi} y_{01}^{\prime \prime} g\left(x_{01}\right)}\right)\left(\frac{h(x)}{g(x)}\right)_{x-r_{11}}^{\prime} .
$$

We see that $\omega^{\prime}\left(y_{00}\right)\left(\partial y^{\prime} / \partial x\right)_{10}$ changes sign at $x=x_{\text {m:1x }}$, so in situation (c) we have

- either an attractor (A) and a saddle (S) with $\mathscr{F}<1$ if $\epsilon y_{0} h\left(x_{\max }\right)<0$ (cf. eq. (2.4)),

- or a repeller $(\mathrm{R})$ and a saddle $(\mathrm{S})$ with $\mathscr{I}>1$ if $\epsilon y_{0} h\left(x_{\max }\right)>0$.

In situation (b) the single FP2 has one eigenvalue equal to 1 , and the other smaller or larger than 1, again depending on the sign of $\epsilon y_{0} h\left(x_{\max }\right)$. Here the saddle-node bifurcation takes place.

The case that $h(x) / g(x)$ has a minimum instead of a maximum can be treated analogously.

As an example of this bifurcation we consider the map (1.3) with the choices

$$
\omega(y)=y, \quad g(x)=\cos (2 \pi x), \quad h(x)=\cos (4 \pi x) .
$$

that is,

$$
T:\left\{\begin{array}{c}
x^{\prime}=x+y \quad(\bmod 1), \\
y^{\prime}=\frac{y+\frac{K}{2 \pi} \cos \left(2 \pi x^{\prime}\right)}{1-\epsilon y \cos \left(4 \pi x^{\prime}\right)} .
\end{array}\right.
$$


We then have

$$
\frac{h(x)}{g(x)}=2 \cos (2 \pi x)-\frac{1}{\cos (2 \pi x)}
$$

and

$$
\left(\frac{h(x)}{g(x)}\right)^{\prime}=-2 \pi \sin (2 \pi x)\left(2+\frac{1}{\cos ^{2}(2 \pi x)}\right),
$$

so the maxima and minima of $h(x) / g(x)$ are at $x=0(\bmod 1)$ and at $x=\frac{1}{2}$ $(\bmod 1)$, respectively.

Taking $y_{0}=1$ we have the bifurcation at $x_{\max }=0$ at $K=-2 \pi \epsilon$ and the bifurcation at $x_{\min }=\frac{1}{2}$ at $K=2 \pi \epsilon$. In fig. 2 the bifurcation at $x=0$ is illustrated.
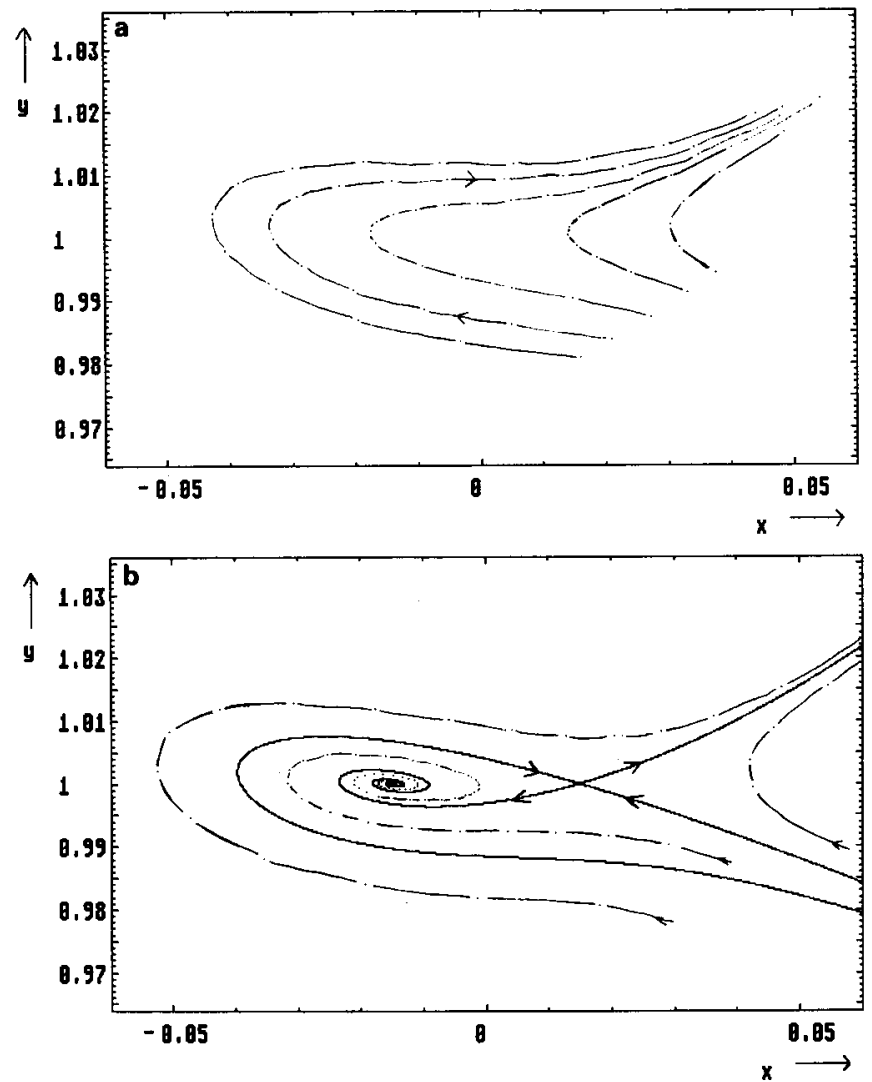

Fig. 2. Saddle-node bifurcation. The figures are phase portraits of the example map (3.4). (a) At $K=0.32$ and $\epsilon=-0.05$. Here $K$ is slightly below the bifurcation value $-2 \pi \epsilon$. This is the situation (a) in the text where there are no FP2 in the neighbourhood of $(0,1)$. (b) At $K=0.31$ and $\epsilon=-0.05$. Here $K$ is slightly above the bifurcation value $-2 \pi \epsilon$. This is the situation (c) in the text. There are two FP2, an attractor (A) and a saddle (S) with $\mathscr{f}<1$. 


\subsection{Transcritical bifurcation}

If there is a FP1 at $x=x_{1}$, which is not a maximum or a minimum of $h(x) / g(x)$ we have for positive $(h(x) / g(x))_{x-v_{1}}^{\prime}$ one of the situations depicted in fig. 3. At $x=x_{1}$ we have $h\left(x_{1}\right)=g\left(x_{1}\right)=0$ and the function $h(x) / g(x)$ has the value

$$
\lim _{x \rightarrow x_{1}} \frac{h(x)}{g(x)}=\frac{h^{\prime}\left(x_{1}\right)}{g^{\prime}\left(x_{1}\right)} .
$$

The three situations to be distinguished are

(a) $-\frac{K}{2 \pi \epsilon} y_{0}^{-2}>\frac{h^{\prime}\left(x_{1}\right)}{g^{\prime}\left(x_{1}\right)}$.

We have a FP1 at $x=x_{1}$ and a FP2 at $x=x_{10}($ a $)>x_{1}$.

(b) $-\frac{K}{2 \pi \epsilon} y_{01}^{-2}=\frac{h^{\prime}\left(x_{1}\right)}{g^{\prime}\left(x_{1}\right)}$.

We only have a fixed point at $x=x_{1}$.

(c) $-\frac{K}{2 \pi \epsilon} y_{0}^{-2}<\frac{h^{\prime}\left(x_{1}\right)}{g^{\prime}\left(x_{1}\right)}$.

We have a FP1 at $x=x_{1}$ and a FP2 at $x=x_{0}(\mathrm{c})<x_{1}$.

The stability of the FP1 $x_{1}$ follows from

$$
\begin{aligned}
\omega^{\prime}\left(y_{0}\right)\left(\frac{\partial y^{\prime}}{\partial x}\right)_{x_{1}} & =\omega^{\prime}\left(y_{0}\right) \frac{K}{2 \pi} g^{\prime}\left(x_{1}\right)+\epsilon y_{11}^{2} h^{\prime}\left(x_{1}\right) \\
& =-\omega^{\prime}\left(y_{0}\right) \epsilon y_{0}^{2} g^{\prime}\left(x_{1}\right)\left(-\frac{K}{2 \pi \epsilon} y_{0}^{2}-\frac{h^{\prime}\left(x_{1}\right)}{g^{\prime}\left(x_{1}\right)}\right) .
\end{aligned}
$$

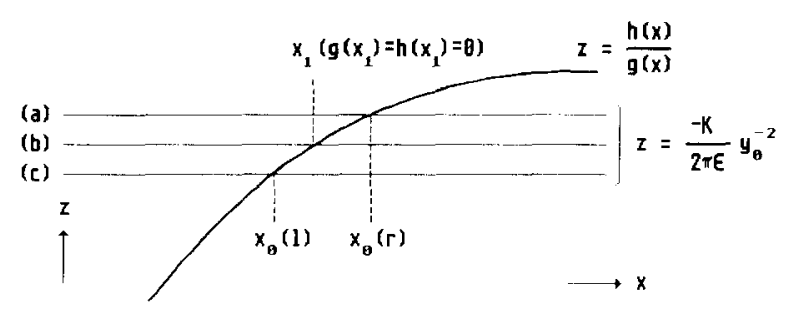

Fig. 3. The function $z=h(x) / g(x)$ near a FP1 (at $x=x_{1}$ ), at which there is neither a maximum nor a minimum. Also drawn are lines $z=-(K / 2 \pi \epsilon) y_{0}{ }^{2}$. The fixed points of type FP2 in situation (a) and (c) are denoted by $x_{11}(1)$ and $x_{11}(\mathrm{r})$, respectively. 
This quantity changes sign at the bifurcation, so the FP1 at $x=x_{1}$ changes from a saddle into a center or vice versa when the FP2 at $x=x_{0}$ passes through the FP1.

On the other hand, the stability of the FP2 $x_{0}$ follows from

$$
\omega^{\prime}\left(y_{0}\right)\left(\frac{\partial y^{\prime}}{\partial x}\right)_{0}=\frac{\omega^{\prime}\left(y_{0}\right) \epsilon y_{0}^{2} g^{\prime}\left(x_{0}\right)}{1-\epsilon y_{0} h\left(x_{0}\right)}\left(\frac{h^{\prime}\left(x_{0}\right)}{g^{\prime}\left(x_{0}\right)}-\frac{h\left(x_{0}\right)}{g\left(x_{0}\right)}\right) .
$$

Near $x=x_{1}$ we make the expansions

$$
\begin{aligned}
-\frac{K}{2 \pi \epsilon} y_{0}^{-2} & =\frac{h\left(x_{0}\right)}{g\left(x_{0}\right)} \\
& =\frac{h^{\prime}\left(x_{1}\right)}{g^{\prime}\left(x_{1}\right)}+\left(\frac{h^{\prime \prime}\left(x_{1}\right) g^{\prime}\left(x_{1}\right)-h^{\prime}\left(x_{1}\right) g^{\prime \prime}\left(x_{1}\right)}{2\left[g^{\prime}\left(x_{1}\right)\right]^{2}}\right)\left(x_{0}-x_{1}\right)+\text { h.o.t. },
\end{aligned}
$$

and

$$
\frac{h^{\prime}\left(x_{0}\right)}{g^{\prime}\left(x_{0}\right)}=\frac{h^{\prime}\left(x_{1}\right)}{g^{\prime}\left(x_{1}\right)}+\left(\frac{h^{\prime \prime}\left(x_{1}\right) g^{\prime}\left(x_{1}\right)-h^{\prime}\left(x_{1}\right) g^{\prime \prime}\left(x_{1}\right)}{\left[g^{\prime}\left(x_{1}\right)\right]^{2}}\right)\left(x_{0}-x_{1}\right)+\text { h.o.t. ; }
$$

so close to the bifurcation (situation (b)) eq. (3.9) can be written as

$$
\omega^{\prime}\left(y_{0}\right)\left(\frac{\partial y^{\prime}}{\partial x}\right)_{0}=\omega^{\prime}\left(y_{0}\right) \epsilon y_{0}^{2} g^{\prime}\left(x_{1}\right)\left(-\frac{K}{2 \pi \epsilon} y_{0}^{-2}-\frac{h^{\prime}\left(x_{1}\right)}{g^{\prime}\left(x_{1}\right)}\right) .
$$

This is the same as eq. (3.8) but with the opposite sign.

Furthermore the Jacobian of the FP2 at $x=x_{0}$, which can be expanded as

$$
\mathscr{I}_{0}=1+2 \epsilon y_{0} h^{\prime}\left(x_{1}\right)\left(x_{0}-x_{1}\right)+\text { h.o.t. },
$$

changes from values $\mathscr{F}<1$ to $\mathscr{J}>1$ at the bifurcation.

Resuming the foregoing considerations we conclude that in going from situation (a) to situation (c):

- either a center $(\mathrm{C})$ and a saddle $(\mathrm{S})$ with $\mathscr{F}>1$ change into a saddle with $\mathscr{J}=1(\mathrm{~S})$ and an attractor (A) or vice versa,

- or a center (C) and a saddle (S) with $\mathscr{F}<1$ change into a saddle with $\mathscr{J}=1(\mathrm{~S})$ and a repeller $(\mathrm{R})$ or vice versa.

As an example of this bifurcation we consider the map (1.3) with the choices

$$
\omega(y)=y, \quad g(x)=\sin (2 \pi x)-\frac{1}{2}, \quad h(x)=\sin \left[2 \pi\left(x-\frac{1}{12}\right)\right],
$$


that is,

$$
T:\left\{\begin{array}{l}
x^{\prime}=x+y \quad(\bmod 1), \\
y^{\prime}=\frac{y+\frac{K}{2 \pi} \sin \left(2 \pi x^{\prime}\right)-\frac{1}{2}}{1-\epsilon y \sin \left[2 \pi\left(x^{\prime}-\frac{1}{12}\right)\right]} .
\end{array}\right.
$$

We then have $x_{1}=\frac{1}{12}(\bmod 1)$, so

$$
\lim _{x \rightarrow x_{1}} \frac{h(x)}{g(x)}=\frac{2}{3} \sqrt{3}
$$

and

$$
\lim _{x \rightarrow x_{1}}\left(\frac{h(x)}{g(x)}\right)^{\prime}=\frac{2}{3} \pi .
$$

Taking $y_{0}=1$ we have the bifurcation at

$$
K=-\frac{1}{3} \pi \sqrt{3} \epsilon
$$

In fig. 4 this bifurcation is illustrated.

\subsection{Rimmer bifurcation}

If there is a FP1 at $x=x_{1}$, at which there is a maximum or a minimum of $h(x) / g(x)$ we have a situation as depicted in fig. 5 (for a maximum). At $x=x_{1}$ the function $h(x) / g(x)$ has again the value

$$
\lim _{x \rightarrow x_{1}} \frac{h(x)}{g(x)}=\frac{h^{\prime}\left(x_{1}\right)}{g^{\prime}\left(x_{1}\right)}
$$

and the three situations to be distinguished are the same as those of the previous subsection, namely:
(a) $-\frac{K}{2 \pi \epsilon} y_{0}^{-2}>\frac{h^{\prime}\left(x_{1}\right)}{g^{\prime}\left(x_{1}\right)}$.
(b) $-\frac{K}{2 \pi \epsilon} y_{0}^{-2}=\frac{h^{\prime}\left(x_{1}\right)}{g^{\prime}\left(x_{1}\right)}$,
(c) $-\frac{K}{2 \pi \epsilon} y_{0}^{-2}<\frac{h^{\prime}\left(x_{1}\right)}{g^{\prime}\left(x_{1}\right)}$. 

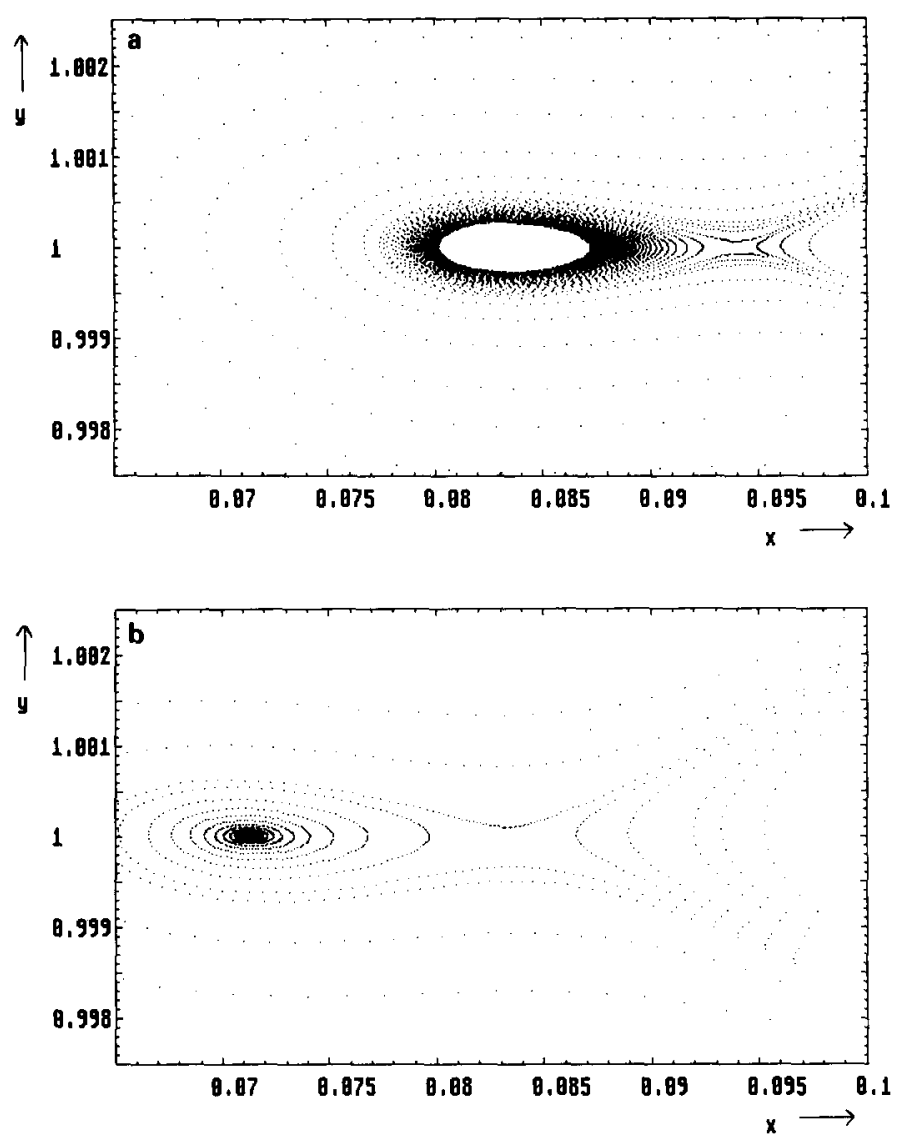

Fig. 4. Transcritical bifurcation. The figures are phase portraits of the example map (3.15). (a) At $K=-0.37$ and $\epsilon=0.05$. Here $K$ is slightly below the bifurcation value $-\frac{4}{3} \pi \sqrt{3} \epsilon$. We have an elliptic fixed point at $x=\frac{1}{12}$ and a saddle with $\mathscr{J}>1$. (b) At $K=-0.355$ and $\epsilon=0.05$. Here $K$ is slightly above the bifurcation value $-\frac{4}{3} \pi \sqrt{3} \epsilon$. We have a saddle with $\mathscr{J}=1$ at $x=\frac{1}{12}$ and an attractor.

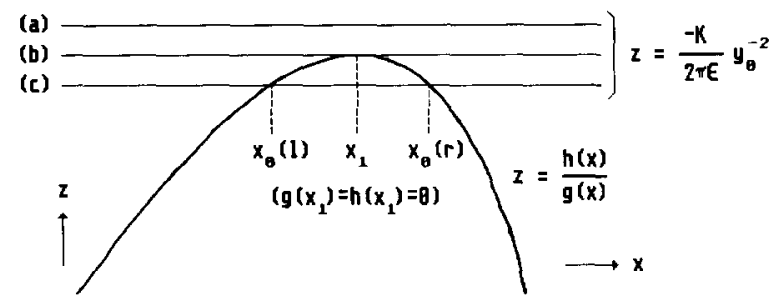

Fig. 5. The function $z=h(x) / g(x)$ near a maximum at $x=x_{1}$, which is a fixed point of type FP1. Also drawn are lines $z=-(K / 2 \pi \epsilon) y_{0}^{-2}$. The two fixed points of type FP2, $x_{0}(1)$ and $x_{0}(\mathrm{r})$, are born from the FP1 (situation (b)) as we go from (a) to (c). 
In situations (a) and (b) we only have a FP1 at $x=x_{1}$, while in situation (c) we have two FP2 at both sides of $x_{1}$ as well.

The stability of the FPl $x_{1}$ is again described by eq. (3.8), which reads

$$
\omega^{\prime}\left(y_{0}\right)\left(\frac{\partial y^{\prime}}{\partial x}\right)_{x_{1}}=-\omega^{\prime}\left(y_{0}\right) \epsilon y_{0}^{2} g^{\prime}\left(x_{1}\right)\left(-\frac{K}{2 \pi \epsilon} y_{0}^{2}-\frac{h^{\prime}\left(x_{1}\right)}{g^{\prime}\left(x_{1}\right)}\right) .
$$

As already mentioned, this quantity changes sign at the bifurcation, so the FP1 at $x=x_{1}$ changes from a saddle into a center or vice versa here.

On the other hand, the stability of the FP2 $x_{0}$ is described by eq. (3.9), reading

$$
\omega^{\prime}\left(y_{0}\right)\left(\frac{\partial y^{\prime}}{\partial x}\right)_{0}=\frac{\omega^{\prime}\left(y_{0}\right) \epsilon y_{0}^{2} g^{\prime}\left(x_{0}\right)}{1-\epsilon y_{01} h\left(x_{01}\right)}\left(\frac{h^{\prime}\left(x_{0}\right)}{g^{\prime}\left(x_{0}\right)}-\frac{h\left(x_{0}\right)}{g\left(x_{01}\right)}\right) \text {. }
$$

Our task is again to expand $h\left(x_{0}\right) / g\left(x_{0}\right)$ and $h^{\prime}\left(x_{01}\right) / g^{\prime}\left(x_{01}\right)$ in the neighbourhood of $x=x_{1}$, as in eqs. (3.10) and (3.11). Only now we must do it up to second order in $\left(x_{0}-x_{1}\right)$, since we are dealing with an extremum in this case. We obtain

$$
\begin{aligned}
-\frac{K}{2 \pi \epsilon} y_{0 \prime}^{\prime 2} & =\frac{h\left(x_{0}\right)}{g\left(x_{0}\right)} \\
& =\frac{h^{\prime}\left(x_{1}\right)}{g^{\prime}\left(x_{1}\right)}+\left(\frac{h^{\prime \prime \prime}\left(x_{1}\right) g^{\prime}\left(x_{1}\right)-h^{\prime}\left(x_{1}\right) g^{\prime \prime \prime}\left(x_{1}\right)}{6\left[g^{\prime}\left(x_{1}\right)\right]^{2}}\right)\left(x_{0}-x_{1}\right)^{2}+\text { h.o.t. }
\end{aligned}
$$

and

$$
\frac{h^{\prime}\left(x_{0}\right)}{g^{\prime}\left(x_{0}\right)}=\frac{h^{\prime}\left(x_{1}\right)}{g^{\prime}\left(x_{1}\right)}+\left(\frac{h^{\prime \prime \prime}\left(x_{1}\right) g^{\prime}\left(x_{1}\right)-h^{\prime}\left(x_{1}\right) g^{\prime \prime \prime}\left(x_{1}\right)}{2\left[\left.g^{\prime}\left(x_{1}\right)\right|^{2}\right.}\right)\left(x_{11}-x_{1}\right)^{2}+\text { h.o.t. ; }
$$

so close to the bifurcation (situation (b)) eq. (3.21) can be written as

$$
\omega^{\prime}\left(y_{0}\right)\left(\frac{\partial y^{\prime}}{\partial x}\right)_{0}=2 \omega^{\prime}\left(y_{0}\right) \epsilon y_{0}^{2} g^{\prime}\left(x_{1}\right)\left(-\frac{K}{2 \pi \epsilon} y_{0}^{2}-\frac{h^{\prime}\left(x_{1}\right)}{g^{\prime}\left(x_{1}\right)}\right) \text {. }
$$

This is the same as eq. (3.20) multiplied by -2 .

Furthermore we have the expansion of the Jacobian of the FP2 of the previous subsection, reading

$$
\mathscr{J}_{0}=1+2 \epsilon y_{0} h^{\prime}\left(x_{1}\right)\left(x_{0}-x_{1}\right)+\text { h.o.t. }
$$


The Jacobian is seen to change sign at $x=x_{1}$, so one of the two FP2 has $\mathscr{J}<1$ while the other has $\mathscr{J}>1$.

Resuming the foregoing considerations we conclude that in going from situation (a) to situation (c):

- either a center $(C)$ changes into a saddle (S) with $\mathscr{F}=1$, giving birth to an attractor (A) and a repeller (R),

- or a saddle with $\mathscr{J}=1$ (S) changes into a center (C), giving birth to a saddle (S) with $\mathscr{J}<1$ and a saddle (S) with $\mathscr{J}>1$.

These two possibilities are schematically illustrated in fig. 6 below.

As an example of this bifurcation we consider the map (1.3) with the choices

$$
\omega(y)=y, \quad g(x)=\sin (2 \pi x), \quad h(x)=\sin (4 \pi x),
$$

that is,

$$
T:\left\{\begin{array}{c}
x^{\prime}=x+y \quad(\bmod 1), \\
y^{\prime}=\frac{y+\frac{K}{2 \pi} \sin \left(2 \pi x^{\prime}\right)}{1-\epsilon y \sin \left(4 \pi x^{\prime}\right)} .
\end{array}\right.
$$

We then simply have

$$
\frac{h(x)}{g(x)}=2 \cos (2 \pi x)
$$

so the maxima and minima of $h(x) / g(x)$ are at the FP1 $x=0(\bmod 1)$ and $x=\frac{1}{2}$ $(\bmod 1)$, respectively.

Taking $y_{0}=1$ we have the bifurcation of $x_{\max }=0$ at $K=-4 \pi \epsilon$ and the bifurcation at $x_{\min }=\frac{1}{2}$ at $K=4 \pi \epsilon$. In figs. 7 and 8 the two possible bifurcations at $x=0$ are illustrated.

It should be noted that in the case of a reversible map with a symmetric fixed point, e.g. the map (1.3) with $g(x)$ and $h(x)$ both odd, the two newly born fixed points move off the symmetry lines. This generalizes the bifurcation in
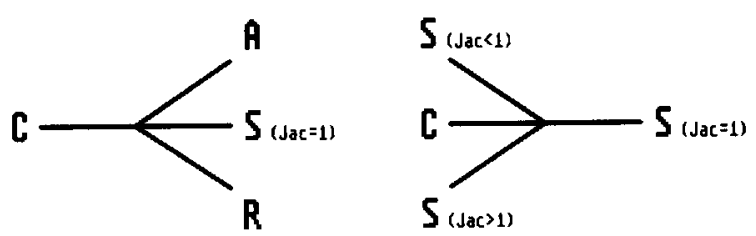

Fig. 6. Schematical representation of the Rimmer bifurcation. A center is indicated by (C), a saddle by $(S)$, an attractor by $(A)$ and a repeller by $(R)$. 

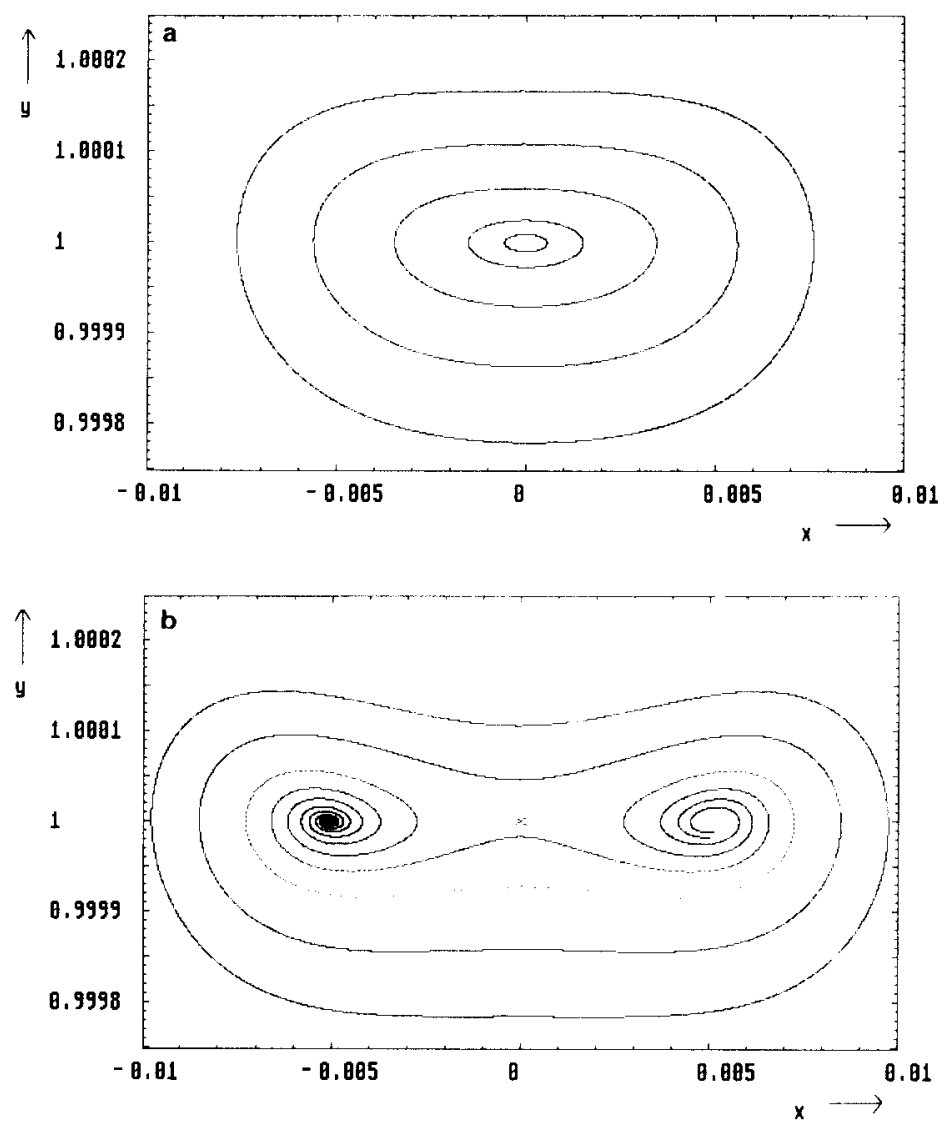

Fig. 7. Rimmer bifurcation. The figures are phase portraits of the example map (3.27). (a) At $K=-0.6286$ and $\epsilon=0.05$. Here $K$ is slightly below the bifurcation value $-4 \pi \epsilon$. We have a center at $x=0$. (b) At $K=-0.628$ and $\epsilon=0.05$. Here $K$ is slightly above the bifurcation value $-4 \pi \epsilon$. We have a saddle with $\mathscr{f}=1$ and an attractor and a repeller. In both figures (a) and (b) the fixed points are surrounded by KAM curves.

reversible area-preserving maps studied by Rimmer [11-13] to the case of reversible non-area-preserving maps. However, the bifurcation depicted in figs. 6-8 can also occur under more general conditions, if the FP1 is an asymmetric fixed point of a reversible mapping, or even if the mapping is not reversible. For convenience we will call this a Rimmer bifurcation as well. In the next section the conditions for these more general Rimmer bifurcations will be investigated and related to the concept of local reversibility introduced in ref. [14].

The Rimmer bifurcations have been observed earlier in conservative systems, e.g. in the appearance of two period-6 orbits in the Hénon map [11], and 

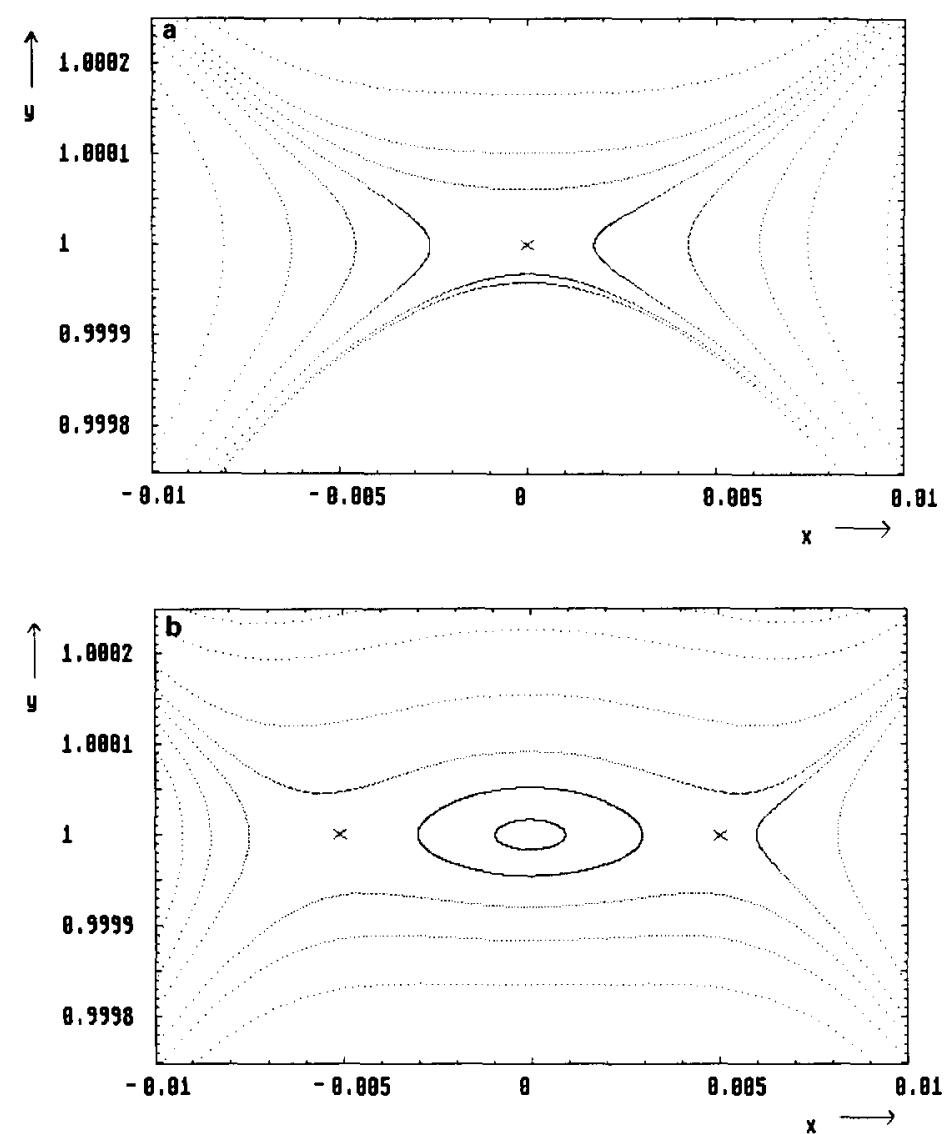

Fig. 8. Rimmer bifurcation. The figures are phase portraits of the example map (3.27). (a) At $K=0.6286$ and $\epsilon=-0.05$. Here $K$ is slightly above the bifurcation value $-4 \pi \epsilon$. We have a saddle with $\mathscr{J}=1$ at $x=0$. (b) At $K=0.628$ and $\epsilon=-0.05$. Here $K$ is slightly below the bifurcation value $-4 \pi \epsilon$. We have a center at $x=0$, and two saddles, one with $\mathscr{I}<1$ and the other with $\mathscr{J}>1$. The center in (b) is surrounded by KAM curves.

in period-2 orbits in a Poincaré section associated with a set of reversible non-Hamiltonian differential equations arising from laser physics $[4,5]$. In the second case dissipative features including the appearance of an attractor and a repeller have been observed.

\subsection{Dissipative features}

In connection with the above scenarios it should be mentioned that fixed points of type FP2 can only occur under certain conditions. For instance when we consider $g(x)$ and $h(x)$ such that $h(x) / g(x)$ has a well-defined maximum in $[0,1]$, it is clear that FP2's at a definite $y_{0} \neq 0, \omega\left(y_{0}\right) \in Z$, can only occur for 
large enough values of $\epsilon / K$. This means that when we start from the conservative mapping (1.3) with $\epsilon=0, K \neq 0$, and perturb the mapping into a nonconservative mapping with $\epsilon \neq 0, K \neq 0$, the FP2's will not occur for sufficiently small $\epsilon$.

In the special case that both $g(x)$ and $h(x)$ are odd, the mapping is reversible and moreover the FPl's are symmetric fixed points. This implies in particular that KAM curves may be observed in the neighbourhood of these fixed points, such as e.g. depicted in fig. 7a. Therefore, the non-conservative mapping (1.3) with $\epsilon \neq 0$ wil behave like a conservative system around the fixed point for sufficiently small $\epsilon$. Only at a relatively large value of $|\epsilon|$, such as e.g. the values $\epsilon= \pm K / 4 \pi$, asymmetric fixed points with $\mathscr{I} \neq 1$ will be born, c.f. fig. $7 b$. in which the dissipative features are clearly visible.

\section{Local reversibility}

In the present section we briefly expose the concept of local reversibility [14] and we discuss the implications concerning the various bifurcation scenarios described above. One can ask oneself under which conditions these can occur when one is studying the fixed points of some mapping of the plane.

A useful tool is then a local reversibility analysis, that is, one figures out to what extent the map can be written locally near a fixed point as the product of two involutions $W$ and $V$, which leave the fixed point invariant,

$$
T=W V
$$

where the involution $W$ is taken to be

$$
W=S^{-1} U S
$$

$S$ being an arbitrary coordinate transformation and $U$ being the involution

$$
U:\left\{\begin{array}{l}
x^{\prime}=x \\
y^{\prime}=-y
\end{array}\right.
$$

This way to write $W$ implies hardly any loss of generality since locally such as $S$ exists, namely $S=U+W$, provided that $U+W$ is locally invertible.

For this purpose one expands the mapping near a fixed point $\left(x_{1}, y_{1}\right)$ in powers of $w=x-x_{1}$ and $z=y-y_{1}$, up to a certain order,

$$
T:\left\{\begin{array}{l}
w^{\prime}=A w+B z+E w^{2}+F w z+G z^{2}+L w^{3}+M w^{2} z+N w z^{2}+O z^{3}+\cdots \\
z^{\prime}=C w+D z+H w^{2}+J w z+K z^{2}+P w^{3}+Q w^{2} z+R w z^{2}+S z^{3}+\cdots
\end{array}\right.
$$


Next one finds out what the necessary conditions on the coefficients $A, B, \ldots$ are under which one is able to find an involution $V$ and a coordinate transformation $S$, such that the relation

$$
S T=U S V
$$

is satisfied up to $n$th order in $w$ and $z$. If the mapping meets these conditions it is called locally reversible up to order $n$ around $\left(x_{1}, y_{1}\right)$.

For instance, for fixed points that lie on a symmetry line in a reversible map we obviously have local reversibility up to order $\infty$.

The concept of local reversibility can be used as a negative criterion to show that a given mapping is not reversible. Consider e.g. the situation that $\left(x_{1}, y_{1}\right)$ is a fixed point such that it cannot be transformed into any other of the fixed points by an involution. This is in particular the case when the eigenvalues of the linearized mapping at any fixed point are different from the inverse eigenvalues of $\left(x_{1}, y_{1}\right)$. Then $\left(x_{1}, y_{1}\right)$ should be a symmetric fixed point if the mapping is to be reversible. Therefore, if in that case local reversibility is not satisfied at any finite order, it can be concluded that the mapping is not reversible, see ref. [14] for an example related to the map (1.3).

In table I, which is taken from ref. [14], we list the conditions for local reversibility up to third order. The first order condition, which is not in the table, is simply

$$
\mathscr{J}\left(x_{1}, y_{1}\right)=1 .
$$

Table I

Necessary conditions for local reversibility of a mapping $L$ given by the expansion (4.4), after a linear transformation to one of the Jordan normal forms. The first column gives the Jordan normal form of the linear part of $L$ in the various cases. In the second column the necessary conditions at second order are specified. The third order conditions are given in the third column. This table is taken from ref. [14].

\begin{tabular}{llc}
\hline Normal form & Order 2 & Order 3 \\
\hline$\left(\begin{array}{cc}A & 0 \\
0 & A^{-1}\end{array}\right)$ & no conditions & $\begin{array}{c}(A-1)\left(A R+A^{-1} M-F J-2 G H\right) \\
\end{array}$ \\
& & $+E F\left(A^{-2}-2 A^{-1}\right)+J K\left(2 A^{2}-A^{3}\right)=0$ \\
$\left(\begin{array}{cc}1 & B \\
0 & 1\end{array}\right)$ & either & if $H=0$ then \\
& $J+2 E-2 B H=0$ & $3 B^{2} P-B Q-3 B L+2 E K+E F+6 B E^{2}=0$ \\
& or & $6 B^{2} P-2 B Q-6 B L+2 E K$ \\
& $H=0$ & $+6 B E^{2}+B E J+2 B J^{2}-F J-J K=0$ \\
$\left(\begin{array}{cc}-1 & B \\
0 & -1\end{array}\right)$ & no conditions & $12 B P+4 Q+12 L+2 J^{2}+2 E J$ \\
& & $+22 B E H+10 B^{2} H^{2}+10 F H$ \\
& & $+11 B H J+4 H K+12 E^{2}=0$ \\
\hline
\end{tabular}


The three rows of the table thus correspond to the three possible Jordan normal forms that the linear part of the expanded mapping can be brought into. For a more extended discussion of table I we refer to ref. [14].

It may be concluded that the saddle-node bifurcations of subsection (3.1) correspond to zero-order local reversibility at the fixed points. Apart from that we only have to consider the local reversibility conditions at the FPl's. In the following we will show that in order to have the Rimmer type of bifurcation at a FP1 type fixed point the local reversibility should be satisfied up to second order. On the other hand, in order to have the transcritical type of bifurcation only first order local reversibility is required. This requirement is automatically satisfied for a FP1 though.

\subsection{Local analysis at the FPI}

In order to work out the conditions for local reversibility, we expand the map (1.3) around a FP1 up to third order in the local coordinates $w=x-x_{1}$, $z=y-y_{1}$, i.e.

$$
\begin{aligned}
& \omega(y)=b z+\omega_{2} z^{2}+\omega_{3} z^{3}, \\
& g(x)=g_{1} w+g_{2} w^{2}+g_{3} w^{3}, \\
& h(x)=h_{1} w+h_{2} w^{2}+h_{3} w^{3} .
\end{aligned}
$$

In this way we obtain a local map of which the linear part is given by

$$
\left(\begin{array}{l}
w^{\prime} \\
z^{\prime}
\end{array}\right)=\left(\begin{array}{cc}
1 & b \\
\lambda & 1+b \lambda
\end{array}\right)\left(\begin{array}{l}
w \\
z
\end{array}\right)
$$

with

$$
\lambda=\frac{K}{2 \pi} g_{1}+\epsilon y_{0}^{2} h_{1} .
$$

We refer to appendix $A$ for some details of the derivation of this form and also of the quadratic and cubic parts, i.e. the higher order coefficients $E, \ldots, S$.

The eigenvalue equation which follows from (4.10) is

$$
(A-1)^{2}=A b \lambda,
$$

where $A$ denotes the eigenvalue. The FP1 is elliptic for $|\operatorname{Tr}|<2$, that is

$$
-4<\lambda b<0
$$

and it is hyperbolic if $\lambda b<-4$ or $\lambda b>0$. 
At $\lambda b=-4$ we have the single eigenvalue $A=-1$, meaning that the elliptic fixed point period-doubles here. At $\lambda b=0$ we have the single eigenvalue $A=+1$. This is precisely the situation for which a bifurcation of the transcritical type or the Rimmer type as treated in subsections (3.2) and (3.3) could occur. The linear matrix in this case reduces to

$$
\left(\begin{array}{ll}
1 & b \\
0 & 1
\end{array}\right)
$$

i.e. the second Jordan normal form of table I. (The period-doubling case with $\lambda b=-4$ corresponds to the third Jordan normal form of table I.)

\subsection{Second order conditions}

To evaluate the conditions for local reversibility in the case $\lambda=0$ one simply reads the coefficients from the mapping (4.4) (given in appendix $A$, in eqs. (A.7), (A.8)) and works out the conditions given in table 1. One then obtains for second order local reversibility the condition that either

$$
J+2 E-2 B H=2 \epsilon y_{0} h^{\prime}\left(x_{1}\right)=0,
$$

or

$$
H=\epsilon y_{0}^{2} g^{\prime}\left(x_{1}\right)\left(\frac{h(x)}{g(x)}\right)_{x=x}^{\prime}=0 .
$$

The first condition, which is also the condition for second order local measure preservation [14] is in general not satisfied. It may only give rise to a bifurcation at a FP1 in the case that $h^{\prime}\left(x_{1}\right)=g^{\prime}\left(x_{1}\right)=0$, which we will not investigate in detail.

The second condition states though that the FP1 $\left(x_{1}, y_{1}\right)$ must be at an extremum of $h(x) / g(x)$, which is exactly the requirement for a Rimmer bifurcation to occur.

Thus the conditions $\lambda=0, H=0$ are the conditions for a Rimmer bifurcation in the map (1.3). These conditions hold more generally for any mapping of the form (4.4), with general coefficients, with a linear part given by

$$
\left(\begin{array}{cc}
1 & b \\
\lambda & 1+\lambda b
\end{array}\right)
$$

that is, for $\lambda=0, H=0$ one can have a bifurcation as schematically depicted in fig. 6. Some details of the derivation of the conditions for the mapping with general coefficients are given in appendix B. 
In the case that $\lambda \neq 0$. that is, either before or after the Rimmer bifurcation takes place, there is no condition on the coefficients for second order local reversibility.

\subsection{Third order conditions}

In the case that $\lambda=0$ the third order conditions can again be evaluated directly from eqs. (A.7), (A.8). For $H=0$ we then find the simple condition

$$
h^{\prime \prime}\left(x_{1}\right)=g^{\prime \prime}\left(x_{1}\right)=0 \text {, }
$$

cf. appendix A, subsection A.2. For $H \neq 0$ there is no condition.

For the case $\lambda \neq 0$ the expansion (4.4) around the FPl in the case of the mapping (1.3) must be transformed such that its linear part takes the first normal form of table $I$.

$$
\left(\begin{array}{ccc}
A & 0 & \\
0 & A & 1
\end{array}\right)
$$

The result of this (see appendix A. subsection A.3) is that the condition for third order local reversibility of table I can be worked out to give

$$
\epsilon y_{11}^{2} g^{\prime}\left(x_{1}\right)\left(\frac{h(x)}{g(x)}\right)_{x, 1}^{\prime}=0 \text {. }
$$

Thus it follows that the third order condition for $\lambda \neq 0$ in the case of a FPl of the mapping (1.3) is automatically implied by the second order condition for $\lambda=0$. This, however, is not generally the case for a mapping of the type (4.4) with general coefficients.

\subsection{Example map}

To check the above results we study the map (1.3) with the choices

$$
\begin{aligned}
& w(y)=y+\omega_{2} y^{2}, \\
& g(x)=\sin (2 \pi x)+\delta \sin ^{2}(2 \pi x), \\
& h(x)=\sin (4 \pi x)+\frac{1}{2} \eta \sin ^{2}\left(4 \pi x^{\prime}\right),
\end{aligned}
$$

that is,

$$
T:\left\{\begin{array}{l}
x^{\prime}=x+y+\omega_{2} y^{2} \quad(\bmod 1), \\
y+\frac{K}{2 \pi} \sin \left(2 \pi x^{\prime}\right)+\delta \sin ^{2}\left(2 \pi x^{\prime}\right) \\
y^{\prime}=\frac{y-\epsilon \sin \left(4 \pi x^{\prime}\right)+\frac{1}{2} \eta \sin ^{2}\left(4 \pi x^{\prime}\right)}{1-\epsilon} .
\end{array}\right.
$$


which is locally reversible up to second order near the FP1

$$
\left(x_{1}, y_{1}\right)=\left(0, \frac{2}{1+\sqrt{1+4 \omega_{2}}}\right) \text {, }
$$

but not to third order if

$$
\begin{aligned}
& \eta \neq 0, \\
& \delta=\eta+\lambda,
\end{aligned}
$$
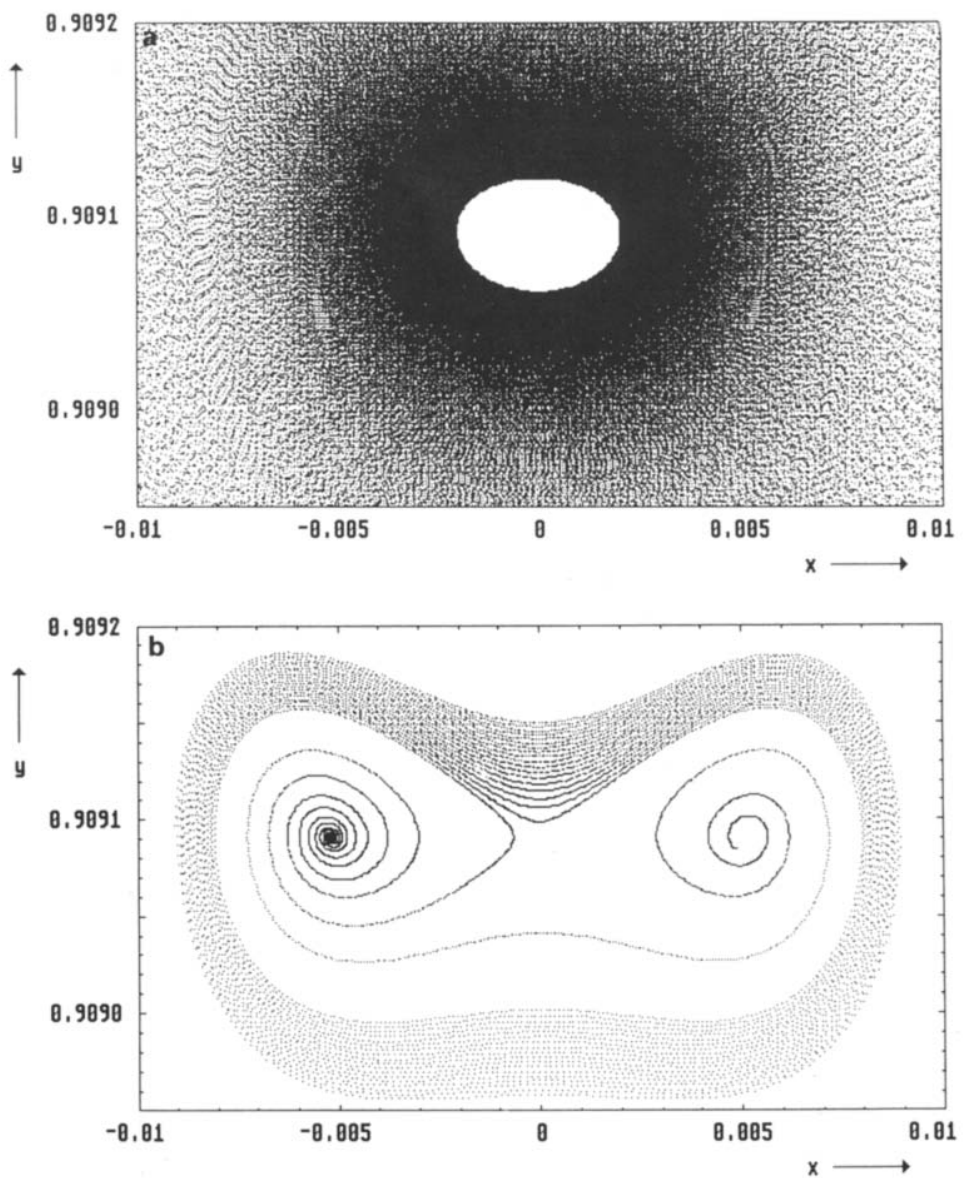

Fig. 9. Rimmer bifurcation in a non-reversible map. The figures are phase portraits of the example map (4.20) with $\omega_{2}=0.11, \eta=0.1$ and $\delta=\eta+\lambda$. (Recall that $\lambda=K+4 \pi \epsilon y_{1}^{2}$.) (a) At $K=$ -0.5195 and $\epsilon=0.05$. Here $\lambda \approx-2.285 \times 10^{-4}$. We have a center at $x=0$. (b) At $K=-0.519$ and $\epsilon=0.05$. Here $\lambda \approx+2.715 \times 10^{-4}$. We have a saddle with $\mathscr{g}=1$ and an attractor and a repeller. (a) represents one single orbit, while (b) represents two orbits. 
cf. eq. (4.17) for the case $\lambda=0$ and eq. (4.18) for the case $\lambda \neq 0$. If we have $\omega_{2} \neq 0$ the mapping is also not globally reversible. As one can check in fig. 9 . where we made phase portraits of the map (4.20), for $\omega_{2}=0.11, \eta=0.1$ and $\delta=\eta+\lambda$, we still have a Rimmer bifurcation of the type sketched in fig. 6 , in which an attractor and a repeller are born from the fixed point $\left(x_{1}, y_{1}\right)=$ $\left(0, \frac{10}{11}\right)$ at $\lambda=0$. In contrast to fig. 7 we see in fig. 9 that the KAM curves around the FP1 do not exist anymore. This is directly related to the violation of local reversibility at the fixed point. A similar picture can be made for the Rimmer bifurcation in the mapping (4.20) for the special case $\omega_{2}=0$. In that case the mapping (4.20) is a reversible mapping, but the FPl is an asymmetric fixed point. In fact, for $\omega_{2} \neq 0$, the mapping (4.20) does not have another fixed point for which the eigenvalues are the inverses of the eigenvalues at $\left(x_{1}, y_{1}\right)$, so then $\left(x_{1}, y_{1}\right)$ should be a symmetric fixed point in order for the map to be reversible. The violation of third order local reversibility shows that the mapping cannot be reversible.

\section{Concluding remarks}

In fig. 10, we present two similar pictures of the map $(4.20)$ in the case that $\delta=\eta$. so here the third order condition for local reversibility is satisfied for $\lambda \neq 0$. (It is not satisfied at $\lambda=0$.) From the figures we may infer that also in this case (with $\omega_{2} \neq 0$ ) the mapping is not reversible. In fact, if the mapping were reversible the FPl would be a symmetric fixed point (and also elliptic for $\lambda<0)$ and in that case one expects to observe surrounding KAM curves [15-17] as in fig. 7, rather than the (extended) chaotic structures.

It turns out that it takes much more time for an orbit to wander away from the elliptic structure it seems to describe when it is started than in the case of fig. 9 , in which the local reversibility is only satisfied up to second order. More generally one might anticipate a relation between the time needed to wander away from the elliptic structure and the order of the local reversibility at the FPl. In the future we hope to give a more detailed analysis of the dynamical aspects of the spiralling of an orbit from a FPl type fixed point.

In fig. 11. finally, we present two phase portraits and the corresponding Rimmer bifurcation in the case of the mapping [18]

$$
T:\left\{\begin{array}{l}
x^{\prime}=(C-y)\left(y^{\prime 2}-2 y^{\prime}+2\right), \\
y^{\prime}=\frac{x}{1+(y+1-C)^{2}} .
\end{array}\right.
$$

This mapping is reversible, i.e. $T=I_{1} I_{2}$ with $I_{1}$ and $I_{2}$ given by 

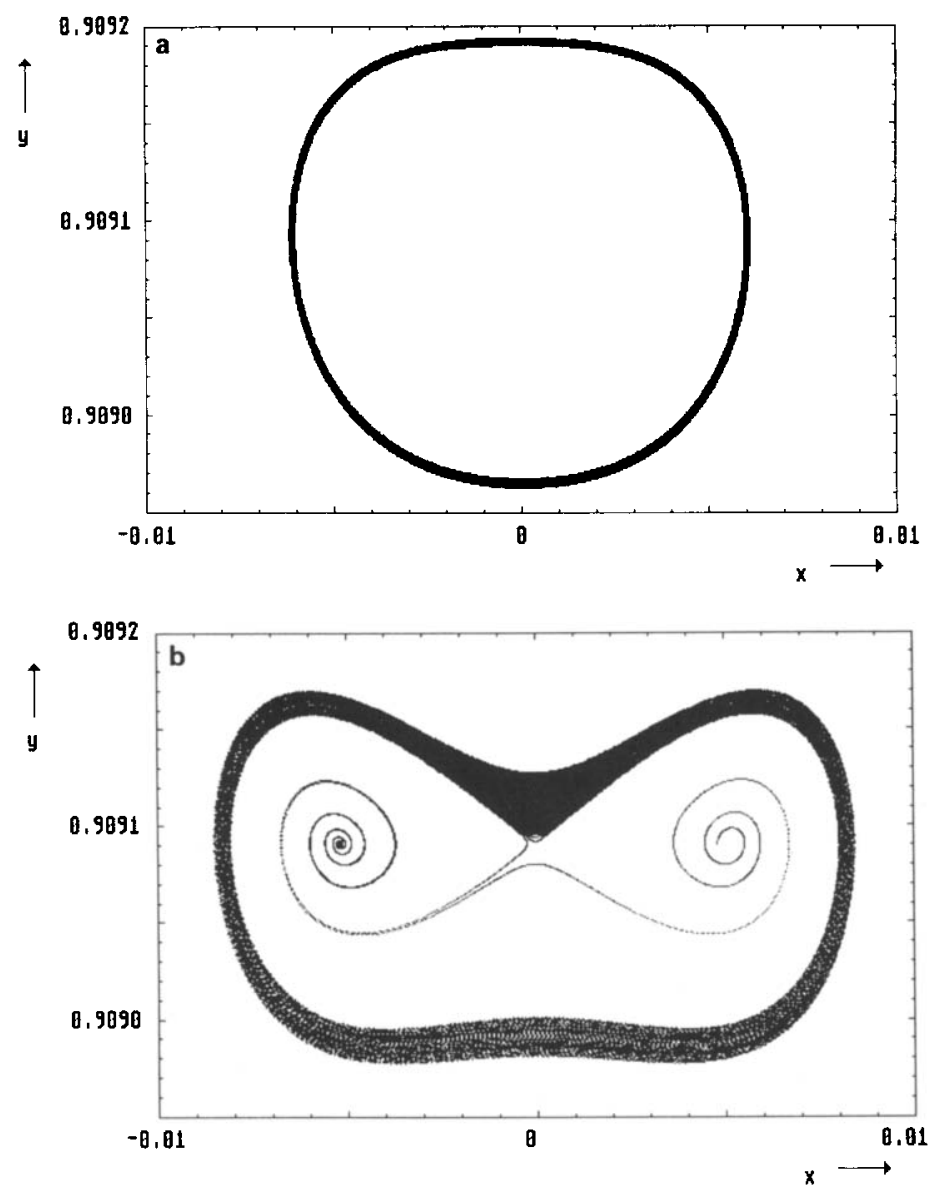

Fig. 10. Rimmer bifurcation in a non-reversible mapping. The figures are phase portraits of the example map (4.20) with $\omega_{2}=0.11$, and $\eta=\delta=0.1$. (Recall that $\lambda=K+4 \pi \epsilon y_{1}^{2}$.) (a) At $K=$ -0.5195 and $\epsilon=0.05$. Here $\lambda \approx-2.285 \times 10^{-4}$. We have a center at $x=0$. (b) At $K=-0.519$ and $\epsilon=0.05$. Here $\lambda \approx-2.285 \times 10^{-4}$. We have a center at $x=0$. (b) At $K=-0.519$ and $\epsilon=0.05$. Here $\lambda \approx+2.715 \times 10^{-4}$. We have a saddle with $\mathscr{J}=1$ and an attractor and a repeller. In both cases we have local reversibility up to order 3 . The surrounding orbit in both figures spirals away much slower from an elliptic structure than in fig. 9, where we had only second order local reversibility. (a) represents one single orbit, while (b) represents two orbits.

$$
I_{1}:\left\{\begin{array}{l}
x^{\prime}=y\left[1+\left(y^{\prime}-1\right)^{2}\right], \\
y^{\prime}=\frac{x}{1+(y-1)^{2}},
\end{array} I_{2}:\left\{\begin{array}{l}
x^{\prime}=x, \\
y^{\prime}=C-y .
\end{array}\right.\right.
$$

In contrast to the mapping (1.3), the mapping (5.1) and the associated involutions do not show any singularities. The pictures close to the bifurcation 

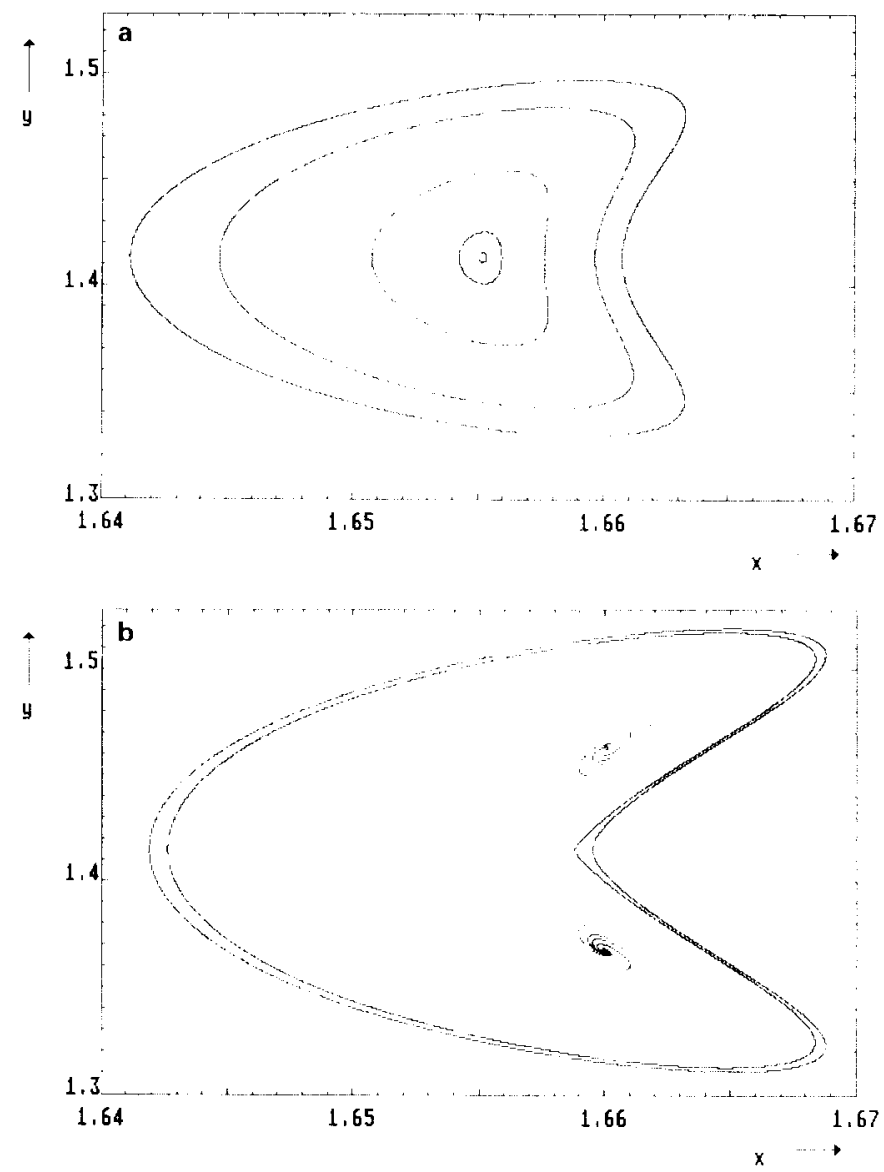

Fig. 11. Rimmer bifurcation in a reversible map. The tigures are phase portrails of the example map (5.1). (a) At $K=2.827$. which is slightly below the bifurcation value $(=2 \sqrt{2}-$ 2.828427125 . . We have a conter at $(2(C-2),(1 / 2)$. (b) At $K=2.83$, which is slightly above the bifurcation value $C=2 \sqrt{2}=2.828427125 \ldots$. We have a saddle with $f=1$ and an attractor and a repeller. In both figures (a) and (b) the fixed points are surrounded by KAM curves.

value $C=2 \sqrt{2}$ at the symmetric FPl $\left(x_{1}, y_{1}\right)=(2(C-2), C / 2)$ are very similar to fig. 7 with KAM type of curves around the symmetric fixed point.

In general, reversible non-area-preserving mappings can be obtained from reversible area-preserving mappings as follows.

Let $T_{\text {AP }}$ be a reversible area-preserving map, with decomposition $T_{\text {AP }}=$ $I_{\mathrm{AP} 1} I_{\mathrm{AP}=}$.

Apply an arbitrary coordinate transformation $S$ on one of the involutions. say on $I_{A P}$, and obtain

$$
T_{\mathrm{NAP}}=S^{-1} I_{\mathrm{AP} !} S I_{\mathrm{AP} Z} .
$$


which will in general not be area preserving. This trick is due to K.M. Pinnow [19]. For example, in (5.1) we have [18]

$$
I_{\mathrm{AP} 1}:\left\{\begin{array}{l}
x^{\prime}=y, \\
y^{\prime}=x
\end{array} \text { and } S:\left\{\begin{array}{l}
x^{\prime}=\frac{x}{1+(y-1)^{2}}, \\
y^{\prime}=y .
\end{array}\right.\right.
$$

Finally, the bifurcation scenarios described above can be reproduced in a phase diagram in control-parameter space, that is, one can indicate for instance the regions in the $K-\epsilon$ plane in which asymmetric fixed points occur. It would be of much interest to investigate also the regions with higher-period asymmetric orbits. This we hope to do in the future.

Another point of interest would be to have a closer look at the cross-over properties of Feigenbaum constants and scaling factors close to the symmetric fixed points at which the asymmetric fixed points are born. Such cross-over properties have been studied in refs. [20,21], in the case of the Hénon map with (constant) Jacobian ranging from 0 to 1 , but here the situation may be slightly more involved.

\section{Acknowledgement}

G.R.W.Q. is grateful to the foundation FOM for supporting his visits to the Institute for Theoretical Physics of the University of Amsterdam.

\section{Appendix A}

\section{Local reversibility analysis}

In this appendix a detailed local reversibility analysis is performed near the FP1 type fixed points of the map (1.3), reading

$$
T:\left\{\begin{array}{l}
x^{\prime}=x+\omega(y) \quad(\bmod 1), \\
y^{\prime}=\frac{y+\frac{K}{2 \pi} g\left(x^{\prime}\right)}{1-\epsilon y h\left(x^{\prime}\right)} .
\end{array}\right.
$$

As before, we denote the FP1 by $\left(x_{1}, y_{1}\right)$.

\section{A.1. Expansion around the FP1}

For the FP1 type fixed points we have $h\left(x_{1}\right)=g\left(x_{1}\right)=0$. Furthermore we have $\omega(y) \in Z$. We now expand around such a fixed point. For this purpose we 
introduce the translated coordinates

$$
\begin{aligned}
& w=x-x_{1} . \\
& z=y-y_{1} .
\end{aligned}
$$

With the expansions

$$
\begin{aligned}
& \omega(y)=b z+\omega_{2} z^{2}+\omega_{3} z^{3} . \\
& g(x)=g_{1} w+g_{2} w^{2}+g_{3} w^{3}, \\
& h(x)=h_{1} w+h_{2} w^{2}+h_{3} w^{3} .
\end{aligned}
$$

we obtain for the mapping up to third order in powers of $w$ and :

$$
\begin{aligned}
& w^{\prime}=w+b z+w_{2} z^{2}+\omega_{3} z^{3} . \\
& z^{\prime}=w \lambda+z(1+b \lambda) \\
& +w^{2}\left[\epsilon y_{11}^{2}\left(-g_{1}{ }^{\prime} g_{2} h_{1}+h_{2}\right)+\lambda\left(\epsilon y_{01} h_{1}+g_{1}{ }^{\prime} g_{2}\right)\right] \\
& +2 w z\left|b \epsilon y_{0}^{2}\left(-g_{1}{ }^{\prime} g_{2} h_{1}+h_{2}\right)+\epsilon y_{11} h_{1}+\lambda b\left(\epsilon y_{10} h_{1}+g_{1}{ }^{\prime} g_{2}\right)\right| \\
& +z^{2}\left[\epsilon b^{2} y_{01}^{2}\left(-g_{1}{ }^{1} g_{2} h_{1}+h_{2}\right)+2 b \epsilon y_{11} h_{1}+\lambda\left(b^{2} \epsilon y_{10} h_{1}+b^{\prime 2} g_{1}{ }^{\prime} g_{2}+\omega_{2}\right)\right] \\
& +w^{3}\left\{\epsilon^{2} y_{1}^{3}\left(-g_{1}{ }^{1} g_{2} h_{1}^{2}+h_{1} h_{2}\right)+\epsilon y_{1}^{2}\left(-g_{1}{ }^{1} g_{3} h_{1}+h_{3}\right)\right. \\
& \left.+\lambda\left|\epsilon^{2} y_{10}^{2} h_{1}^{2}+\epsilon y_{11}\left(g_{1}{ }^{\prime} g_{2} h_{1}+h_{2}\right)+g_{1}{ }^{\prime} g_{3}\right|\right\} \\
& +w^{2} z\left\{3 b \epsilon^{2} y_{11}^{3}\left(-g_{1}{ }^{1} g_{2} h_{1}^{2}+h_{1} h_{2}\right)+3 b \epsilon y_{11}^{2}\left(-g_{1}{ }^{1} g_{3} h_{1}+h_{3}\right)\right. \\
& +2 \epsilon^{2} y_{11}^{2} h_{1}^{2}+2 \epsilon y_{10} h_{2} \\
& +\lambda\left[3 b \epsilon^{2} y_{10}^{2} h_{1}^{2}+3 b \epsilon y_{13}\left(g_{1}{ }^{\prime} g_{2} h_{1}+h_{2}\right)+3 b g_{1}{ }^{\prime} g_{3}+\epsilon h_{1} \mid\right\} \\
& +w z^{2}\left\{3 b^{2} \epsilon^{2} y_{0}^{3}\left(-g_{1}{ }^{1} g_{2} h_{1}^{2}+h_{1} h_{2}\right)+3 b^{2} \epsilon y_{0}^{2}\left(-g_{1}{ }^{1} g_{3} h_{1}+h_{3}\right)\right. \\
& +2 \epsilon y_{11}^{2} \omega_{2}\left(g_{1}{ }^{1} g_{2} h_{1}+h_{2}\right)+4 b \epsilon^{2} y_{10}^{2} h_{1}^{2}+4 b \epsilon y_{01} h_{2}+\epsilon h_{1} \\
& +\lambda\left[3 b^{2} \epsilon^{2} y_{0}^{2} h_{1}^{2}+3 b^{2} \epsilon y_{0}\left(g_{1}^{\prime} g_{2} h_{1}+h_{2}\right)\right. \\
& \left.\left.+3 b^{2} g_{1}{ }^{1} g_{3}+2 b \epsilon h_{1}-2 \omega_{2}\left(\epsilon y_{11} h_{1}+g_{1}{ }^{\prime} g_{2}\right)\right]\right\} \\
& +z^{3}\left[b^{3} \epsilon^{2} y_{0}^{3}\left(-g_{1}{ }^{1} g_{2} h_{1}^{2}+h_{1} h_{2}\right)+b^{3} \epsilon_{(1}{ }^{2}\left(-g_{1}{ }^{1} g_{3} h_{1}+h_{3}\right)\right. \\
& +2 b^{2} \epsilon^{2} y_{0}^{2} h_{1}^{2}+2 b^{2} \epsilon y_{0} h_{2}+b \epsilon h_{1}+2 b \epsilon y_{0}^{2} \omega_{2}\left(-g_{1}{ }^{\prime} g_{2} h_{1}+h_{2}\right)
\end{aligned}
$$




$$
\begin{aligned}
& +2 \epsilon y_{0} \omega_{2} h_{1} \\
& +\lambda\left(b^{3} \epsilon^{2} y_{0}^{2} h_{1}^{2}+b^{3} \epsilon y_{0} g_{1}^{-1} g_{2} h_{1}+b^{3} \epsilon y_{0} h_{2}+b^{3} g_{1}^{-1} g_{3}+b^{2} \epsilon h_{1}\right. \\
& \left.\left.+2 b \epsilon y_{0} \omega_{2} h_{1}+2 b \omega_{2} g_{1}^{-1} g_{2}+\omega_{3}\right)\right]
\end{aligned}
$$

with

$$
\lambda=\frac{K}{2 \pi} g_{1}+\epsilon y_{0}^{2} h_{1}
$$

The linear part of the map in the new coordinates reads

$$
\left(\begin{array}{c}
w^{\prime} \\
z^{\prime}
\end{array}\right)=\left(\begin{array}{cc}
1 & b \\
\lambda & 1+b \lambda
\end{array}\right)\left(\begin{array}{c}
w \\
z
\end{array}\right) \text {. }
$$

The eigenvalue equation is

$$
\lambda A b=(A-1)^{2},
$$

with $A$ the eigenvalue.

A possible Rimmer bifurcation will take place when we have a single eigenvalue $A=1$, that is, when $\lambda=0$. We shall now investigate the local reversibility conditions for $\lambda=0$ and subsequently for $\lambda \neq 0$.

\section{A.2. The case $\lambda=0$}

In this case the linear part is already in the Jacobian normal form

$$
\left(\begin{array}{ll}
1 & b \\
0 & 1
\end{array}\right)
$$

of table I, so we can directly read the coefficients $E, \ldots, S$ from eqs. (A.7), (A.8).

The condition for second order local reversibility is that either

$$
J+2 E-2 B H=0
$$

or

$$
H=0 .
$$

The first condition is also the condition for second order local measure preservation [14]. It evaluates to 


$$
h_{1}=0 \text {, }
$$

which is generally not satisfied. The second condition yields

$$
g_{1}{ }^{1}\left(h_{2} g_{1}-g_{2} h_{1}\right)=0 \text {. }
$$

This is equivalent to

$$
g^{\prime}\left(x_{1}\right)\left(\frac{h(x)}{g(x)}\right)_{x-x_{1}}^{\prime}=0 .
$$

meaning that the FP1 under study must be at an extremum of $h(x) / g(x)$, which is exactly the prerequisite for the Rimmer bifurcation to occur.

The condition for third order local reversibility reads (for $\lambda=0$ )

$$
6 B^{2} P-2 B Q-6 B L+2 E K+B E J+6 B E^{2}+2 B J^{2}-F J-J K=0 .
$$

evaluating to

$$
3 b^{2} \epsilon y_{10}^{3}\left(-2 g_{1}^{-1} g_{2} h_{1} h_{2}+g_{1}^{-2} g_{2}^{2} h_{1}^{2}+h_{2}^{2}\right)-5 b \epsilon y_{0}^{2}\left(g_{1}{ }^{1} g_{2} h_{1}^{2}-h_{1} h_{2}\right)-2 h_{2}=0 .
$$

This simply yields

$$
h^{\prime \prime}\left(x_{1}\right)=g^{\prime \prime}\left(x_{1}\right)=0 \text {. }
$$

\section{A.3. The case $\lambda \neq 0$}

When $\lambda \neq 0$ we have two eigenvalues $A$ and $A^{-1}$, and we can transform the expanded mapping (A.7). (A.8) to the diagonal form

$$
\left(\begin{array}{cc}
A & 0 \\
0 & A^{-1}
\end{array}\right) \text {. }
$$

For this purpose we apply the diagonalizing transformation

$$
\begin{aligned}
& w=\frac{A^{{ }^{\prime 1}} x+y}{1+A^{-1}} . \\
& z=\frac{A^{-1} b^{-1}(1-A)(y-x)}{1+A^{-1}} .
\end{aligned}
$$


Together with the scalings

$$
\begin{aligned}
& \Omega_{2}=\frac{(1-A)^{2} A^{-2} b^{-2}}{1+A^{-1}} \omega_{2}, \\
& \Omega_{3}=\frac{(1-A)^{3} A^{-3} b^{-2}}{\left(1+A^{-1}\right)^{2}} \omega_{3}, \\
& \mathscr{H}_{1}=\frac{\epsilon}{1+A^{-1}} h_{1}, \\
& \mathscr{G}_{2}=g_{1}^{-1} \frac{1}{1+A^{-1}} g_{2}, \\
& \mathscr{H}_{2}=\frac{(1-A)^{-1} \epsilon A b}{1+A^{-1}} g_{1}^{-1}\left(h_{2} g_{1}-g_{2} h_{1}\right), \\
& \mathscr{G}_{3}=g_{1}^{-1} \frac{1}{\left(1+A^{-1}\right)^{2}} g_{3}, \\
& \mathscr{H}_{3}=\frac{(1-A)^{-1} \epsilon A b}{\left(1+A^{-1}\right)^{2}} g_{1}^{-1}\left(h_{3} g_{1}-g_{3} h_{1}\right),
\end{aligned}
$$

we then find the coefficients $E, \ldots, S$ as

$$
\begin{aligned}
E= & \mathscr{H}_{1} A y_{0} \\
& +\frac{1}{1+A}\left[-\mathscr{H}_{2} A y_{0}^{2}+\mathscr{G}_{2} A(A-1)+\Omega_{2} A^{2}\right], \\
F= & \frac{1}{1+A}\left[-2 \mathscr{H}_{2} y_{0}^{2}+2 \mathscr{G}_{2}(A-1)-2 \Omega_{2} A^{2}\right], \\
G= & -\mathscr{H}_{1} A^{-1} y_{0} \\
& +\frac{1}{1+A}\left[-\mathscr{H}_{2} A^{-1} y_{0}^{2}+\mathscr{G}_{2}\left(-A^{-1}+1\right)+\Omega_{2} A^{2}\right], \\
H= & -\mathscr{H}_{1} y_{0} \\
& +\frac{1}{1+A}\left[\mathscr{H}_{2} y_{0}^{2}+\mathscr{G}_{2}(1-A)+\Omega_{2}\right], \\
J= & \frac{1}{1+A}\left[2 \mathscr{H}_{2} A^{-1} y_{0}^{2}+2 \mathscr{G}_{2}\left(A^{-1}-1\right)-2 \Omega_{2}\right],
\end{aligned}
$$




$$
\begin{aligned}
& K=\mathscr{H}_{1} A^{-2} y_{n} \\
& +\frac{1}{1+A}\left[\mathscr{H}_{2} A^{-2} y_{0}^{2}+\mathscr{G}_{2} A^{2}(1-A)+\Omega_{2}\right] \text {. } \\
& L=\mathscr{H}_{1}^{2} A y_{11}^{2} \\
& +\frac{1}{1+A}\left[-\mathscr{H}_{1} \mathscr{H}_{2} A y_{0}^{3}+2 \mathscr{H}_{1} \mathscr{G}_{2} A^{2} y_{0}+2 \mathscr{H}_{1} \mathscr{G}_{2} A^{2} y_{1}\right. \\
& +\mathscr{H}_{1} A^{2} b^{\prime}(A-1) /(A+1)-2 \mathscr{H}_{2} \Omega \Omega_{2} A y_{11}^{2}+\mathscr{H}_{2} A b^{\prime \prime} y_{11}(-A+1) \\
& -\mathscr{H}_{3} A y_{11}^{2}+2 \mathscr{G}_{2} \Omega_{2} A(A-1)+\mathscr{G}_{3} A(A-1)-\Omega_{3} A^{2} b{ }^{\prime} \mathrm{l} \text {. } \\
& M=\mathscr{H}_{1}^{2} y_{0}^{2} \\
& +\frac{1}{1+A}\left(-3 \mathscr{H}_{1} \mathscr{H}_{2} y_{0}^{3}+2 \mathscr{H}_{1} \mathscr{G}_{2} y_{1}(2 A-1)+2 \mathscr{H}_{1} \Omega \Omega_{2} y_{11}\left(-2 A^{2}-1\right)\right. \\
& +\mathscr{H}_{1} b^{1}\left(-A^{3}+2 A^{2}-2 A+1\right) /(A+1)+2 \mathscr{H}_{2} \Omega_{2} y_{11}^{2}(2 A-1) \\
& +\mathscr{H}_{2} b{ }^{1} y_{0}(1-A)-3 \mathscr{H}_{2} y_{11}^{2}+2 \mathscr{G}_{2} \Omega_{2}\left(-2 A^{2}+3 A-1\right) \\
& +3 \mathscr{G}_{3}(A-1)+3 \Omega_{3} A^{2} b{ }^{\prime} \mid \text {. } \\
& N=-\mathscr{H}_{1}^{2} A{ }^{1} y_{11}^{2} \\
& +\frac{1}{1+A}\left[-3 \mathscr{H}_{1} \mathscr{H}_{2} A^{1} y_{11}^{3}+2 \mathscr{H}_{1} \mathscr{G}_{2} y_{01}\left(-2 A^{1}+1\right)+2 \mathscr{H}_{1} \Omega_{2} y_{10}\left(A^{2}+2\right)\right. \\
& +\mathscr{H}_{1} b^{-1}\left(-A^{2}+2 A+A^{1}-2\right) /(A+1)+2 \mathscr{H}_{2} \Omega_{2} y_{11}(2-A) \\
& +\mathscr{H}_{2} b^{\prime} y_{1}\left(1-A^{1}\right)-3 \mathscr{H}_{3} A y_{11}^{2}+2 \mathscr{G}_{2} \Omega_{2}\left(A^{2}-3 A+2\right) \\
& +3 \mathscr{G}_{3}\left(-A^{-1}+1\right)-3 \Omega_{3} A^{2} b^{1} 1 \text {. } \\
& O=-\mathscr{H}_{1}^{2} A^{-2} y_{11}^{2} \\
& +\frac{1}{1+A} 1-\mathscr{H}_{1} \mathscr{H}_{2} A^{2} y_{0}^{3}-2 \mathscr{H}_{1} \mathscr{G}_{2} A^{2} y_{10}-2 \mathscr{H}_{1} \Omega_{2} y_{11} \\
& +\mathscr{H}_{1} b^{\prime \prime}\left(1-A^{1}\right) /(A+1)-2 \mathscr{H}_{2} \Omega_{2} y_{11}^{2}+\mathscr{H}_{2} A^{2} b^{-1} y_{11}(A-1) \\
& \left.-\mathscr{H}_{3} A^{2} y_{0}^{2}+2 \mathscr{G}_{2} \Omega_{2}(A-1)+\mathscr{G}_{3} A^{-2}(A-1)+\Omega_{3} A^{2} b^{1}\right\rfloor \text {, } \\
& P=-\mathscr{H}^{2} y_{11}^{2} \\
& +\frac{1}{1+A}\left[\mathscr{H}_{1} \mathscr{H}_{2} y_{0}^{3}-2 \mathscr{H}_{1} \mathscr{G}_{2} A y_{0}-2 \mathscr{H}_{1} \Omega_{2} A y_{0}\right. \\
& +\mathscr{H}_{1} A b^{-1}(1-A) /(A+1)+2 \mathscr{H}_{2} \Omega_{2} y_{0}^{2}+\mathscr{H}_{2} b^{-1} y_{0}(A-1) \\
& \left.+\mathscr{H}_{3} y_{0}^{2}+2 \mathscr{G}_{2} \Omega_{2}(1-A)+\mathscr{G}_{3}(1-A)-\Omega_{3} b^{-1}\right] \text {. }
\end{aligned}
$$




$$
\begin{aligned}
& Q=-\mathscr{H}_{1}^{2} A^{-1} y_{0}^{2} \\
&+\frac{1}{1+A}\left[3 \mathscr{H}_{1} \mathscr{H}_{2} A^{-1} y_{0}^{3}+2 \mathscr{H}_{1} \mathscr{G}_{2} y_{0}\left(A^{-1}-2\right)+2 \mathscr{H}_{1} \Omega_{2} A^{-1} y_{0}\left(2 A^{2}+1\right)\right. \\
&+\mathscr{H}_{1} b^{-1}\left(A^{2}-2 A-A^{-1}+2\right) /(A+1)+2 \mathscr{H}_{2} \Omega_{2} y_{0}^{2}\left(A^{-1}-2\right) \\
&+\mathscr{H}_{2} b^{-1} y_{0}\left(1-A^{-1}\right)+3 \mathscr{H}_{3} A^{-1} y_{0}^{2}+2 \mathscr{G}_{2} \Omega_{2}\left(2 A+A^{-1}-3\right) \\
&\left.+3 \mathscr{G}_{3}\left(A^{-1}-1\right)+3 \Omega_{3} b^{-1}\right], \\
& R= \mathscr{H}_{1}^{2} A^{-2} y_{0}^{2} \\
&+\frac{1}{1+A^{2}}\left[3 \mathscr{H}_{1} \mathscr{H}_{2} A^{-2} y_{0}^{3}+2 \mathscr{H}_{1} \mathscr{G}_{2} A^{-2} y_{0}(2-A)\right. \\
&+2 \mathscr{H}_{1} \Omega_{2} A^{-1} y_{0}\left(-A^{2}-2\right)+\mathscr{H}_{1} b^{-1}\left(A+2 A^{-1}-A^{-2}-2\right) /(A+1) \\
&+2 \mathscr{H}_{2} \Omega_{2} y_{0}^{2}\left(-2 A^{-1}+1\right)+\mathscr{H}_{2} A^{-2} b^{-1} y_{0}(-A+1)+3 \mathscr{H}_{3} A^{-2} y_{0}^{2} \\
&\left.+2 \mathscr{G}_{2} \Omega_{2}\left(-A-2 A^{-1}+3\right)+3 \mathscr{G}_{3} A^{-2}(1-A)-3 \Omega_{3} b^{-1}\right], \\
& \mathscr{H}_{1}^{2} A^{-3} y_{0}^{2} \\
&+\frac{1}{1+A^{-1}}\left[\mathscr{H}_{1} \mathscr{H}_{2} A^{-3} y_{0}^{3}+2 \mathscr{H}_{1} \mathscr{G}_{2} A^{-3} y_{0}+2 \mathscr{H}_{1} \Omega_{2} A^{-1} y_{0}\right. \\
&+\mathscr{H}_{1} A^{-2} b^{-1}\left(1-A_{3} /(A+1)+2 \mathscr{H}_{2} \Omega_{2} A^{-1} y_{0}^{2}+\mathscr{H}_{2} A^{-3} b^{-1} y_{0}(1-A)\right. \\
&\left.+\mathscr{H}_{3} A^{-3} y_{0}^{2}+2 \mathscr{G}_{2} \Omega_{2}\left(A^{-1}-1\right)+\mathscr{G}_{3} A^{-3}(1-A)+\Omega_{3} b^{-1}\right] . \\
&S .43)
\end{aligned}
$$

The condition for third order local reversibility is in this case

$$
\begin{aligned}
& (A-1)\left(A R+A^{-1} M-F J-2 G H\right)+E F\left(A^{-2}-2 A^{-1}\right) \\
& \quad+J K\left(2 A^{2}-A^{3}\right)=0
\end{aligned}
$$

and it evaluates to

$$
\mathscr{H}_{2}\left[\mathscr{H}_{1} A^{-1} y_{0}^{2}+A^{-1} b^{-1}(1-A)^{2} /(A+1)\right]=0
$$

The numerator in the second term between brackets equals $\lambda$, so with eq. (A.9) we obtain

$$
\mathscr{H}_{2} g_{1}=0
$$


which is once again, surprisingly enough, the Rimmer condition (A.17):

$$
g^{\prime}\left(x_{1}\right)\left(\frac{h(x)}{g(x)}\right)_{x-x_{1}}=0 \text {. }
$$

\section{Appendix B}

Bifurcation analysis for a map with general coefficients

In this appendix we discuss the bifurcation scenarios for a map with a linear part given by

$$
\left(\begin{array}{cc}
1 & b \\
\lambda & 1+b \lambda
\end{array}\right)
$$

and otherwise general coefficients. Up to third order we thus write

$$
\begin{aligned}
& w^{\prime}=w+b z+E w^{2}+F w z+G z^{2}+L w^{3}+M w^{2} z+N w z^{2}+O z^{2} \\
& z^{\prime}=\lambda w^{\prime}+(1+\lambda b) z+H w^{2}+J w z+K z^{2}+P w^{3}+Q w^{2} z+R w z^{2}+S z^{\prime}
\end{aligned}
$$

The origin is a fixed point of this map wit Jacobian equal to 1 . It is elliptic for $-4<\lambda b<0$. and hyperbolic for $\lambda b<-4$ or $\lambda b>0$. At $\lambda b=-4$ the origin period-doubles. We shall concentrate here on the bifurcations that can take place at $\lambda b=0$.

\section{B.1. Fixed points outside the origin}

In order to obtain fixed point solutions outside but close to the origin we do the following. First we solve eq. (B.2) with $w^{\prime}=w$ for $z$. obtaining

$$
z=\frac{-1}{b+F w+M w^{2}}\left[E w^{2}+L w^{3}+(G+N w) z^{2}+O z^{3}\right] .
$$

Resubstitution of this expression into the $z$ 's of the right-hand side then yields up to third order in $w$

$$
z=-b^{-1} E w^{2}+\left(b^{-2} E F-b^{-1} L\right) w^{3}
$$

Next we put this into eq. (B.3) with $z^{\prime}=z$, and we get

$$
\left[\lambda\left(b^{-1} E F-L\right)+P-b^{-1} E J\right] w^{3}+(H-\lambda E) w^{2}+\lambda w=0 .
$$


So the fixed point outside the origin is obtained as

$$
w=\frac{1}{2 \alpha}\left[-H+\lambda E \pm \sqrt{(H-\lambda E)^{2}-4 \lambda \alpha}\right],
$$

with

$$
\alpha=\lambda\left(b^{-1} E F-L\right)+P-b^{-1} E J .
$$

There are two fixed points outside the origin for

$$
4 \lambda \alpha<(H-\lambda E)^{2} .
$$

\section{B.2. Stability analysis}

We shall now examine the stability of the fixed points outside the origin. For this purpose we first determine the Jacobian matrix at these points. With $z=-(E / b) w^{2}+\mathscr{O}\left(w^{3}\right)$ we find up to second order in $w$

$$
\begin{aligned}
& \frac{\partial w^{\prime}}{\partial w}=1+2 E w+\left(3 L-b^{-1} E F\right) w^{2}, \\
& \frac{\partial w^{\prime}}{\partial z}=b+F w+\left(M-2 b^{-1} E G\right) w^{2}, \\
& \frac{\partial z^{\prime}}{\partial w}=\lambda+2 H w+\left(3 P-b^{-1} E J\right) w^{2}, \\
& \frac{\partial z^{\prime}}{\partial z}=1+\lambda b+J w+\left(Q-2 b^{-1} E K\right) w^{2} .
\end{aligned}
$$

The trace and the Jacobian are (up to second order)

$$
\begin{aligned}
\operatorname{Tr}= & 2+\lambda b+(J+2 E) w+\left[3 L+Q-b^{-1} E(F+2 K)\right] w^{2}, \\
\mathscr{J}= & 1+[J+2 E-2 b H+\lambda(2 E b-F)] w \\
& +\left[3 L+Q-b^{-1} E(F+2 K)+3 E J-2 F H-3 b P\right. \\
& \left.+\lambda\left(3 b L-E F-M+2 b^{-1} E G\right)\right] w^{2} .
\end{aligned}
$$

The stability is governed by the quantity $\Phi=\operatorname{Tr}-\mathscr{J}-1$,

$$
\begin{aligned}
\Phi= & \lambda b+[2 b H+\lambda(F-2 E b)] w \\
& +\left[2 F H-3 E J+3 b P+\lambda\left(E F+M-3 b L-2 b^{-1} E G\right)\right] w^{2} .
\end{aligned}
$$


We will call $\Phi$ the stability of the fixed point. Fixed points outside (but close to) the origin are elliptic for $\Phi<0$ and hyperbolic for $\Phi>0$. The origin itself is elliptic for $\lambda b<0$ and hyperbolic for $\lambda b>0$.

\section{B.3. Bifurcations}

When we conside eqs. (B.7), (B.9) for fixed points outside the origin we observe that there are three different situations, each of which we will discuss below.

B.3.1. $H=\ell(1)$

If $\lambda$ is small enough there are always two fixed points, namely

$$
w=\frac{1}{2 \alpha}\left|-H+\lambda E \pm H \sqrt{1-\lambda\left(2 E H^{1}+4 \alpha H^{2}\right)+\lambda^{2} E^{2} H^{2}}\right|
$$

or

$$
\left.w=\frac{1}{2 \alpha} \mid-H+\lambda E \pm\left(H-\lambda E-2 \alpha \lambda H^{\prime}\right)\right]+\left(\lambda^{2}\right)
$$

So for $\lambda=0$ we have the $(+)$ solution passing through the origin while the $(-)$ solution lies outside the origin. More specifically, we have

$$
w=-\frac{\lambda}{H}+\sigma\left(\lambda^{2}\right)
$$

for the $(+)$ solution, and

$$
w=-\frac{H}{\alpha}+c(\lambda)
$$

for the $(-)$ solution.

If we consider the stability of the fixed point that passes through the origin we find in terms of $\lambda$ that

$$
\begin{aligned}
& g=1+(J+2 E-2 b H) \lambda+C\left(\lambda^{2}\right) . \\
& \Phi=-\lambda b+\circlearrowleft\left(\lambda^{2}\right) .
\end{aligned}
$$

Thus when $\lambda b$ passes through 0 from below the origin changes from a center into a saddle (with $\not=1$ of course) while for the fixed point that passes through the origin we have the following possibilities:

- If $J+2 E-2 b H>0$ then the fixed point changes from a saddle with $f<1$ into a repeller. 
- If $J+2 E-2 b H<0$ then the fixed point changes from a saddle with $\mathscr{F}>1$ into an attractor.

These are transcritical types of bifurcations. We have schematically represented them in fig. 12.

Finally there is also the possibility that $J+2 E-2 b H=0$. Then the fixed point outside the origin changes from a saddle with $\mathscr{J}<1$ into an attractor in case the coefficient of $w^{2}$ in eq. (B.15) (with $\lambda=0$ ) is negative. In case this coefficient is positive, the fixed point changes from a saddle with $\mathscr{f}>1$ into a repeller.

B.3.2. $H=\mathscr{O}(\sqrt{|\lambda|})$

In this case we write more specifically

$$
H=\beta \sqrt{|\lambda|}+\mathcal{O}(\lambda),
$$

introducing the coefficient $\beta$.

The fixed points outside the origin exist if

$$
(H-\lambda E)^{2}-4 \alpha \lambda=\beta^{2}|\lambda|-4 \alpha \lambda+\mathscr{O}\left(\lambda^{3 / 2}\right)>0,
$$

that is, if $\beta^{2}>-4 \alpha$ they exist for $\lambda<0$, and if $\beta^{2}>4 \alpha$ they exist for $\lambda>0$.

For small positive $\lambda$ we find for the fixed points outside the origin, provided that $\alpha<\beta^{2} / 4$,

$$
w=\frac{1}{2 \alpha}\left(-\beta \pm \sqrt{\beta^{2}-4 \alpha}\right) \sqrt{\lambda}+\mathscr{O}(\lambda)
$$

For the Jacobian $\mathscr{g}$ and the stability $\Phi$ we obtain in terms of $\lambda$ that

$$
\begin{aligned}
\mathscr{J}= & +\frac{J+2 E}{2 \alpha}\left(-\beta \pm \sqrt{\beta^{2}-4 \alpha}\right) \sqrt{\lambda} \\
& +\left(\frac{-b \beta}{\alpha}\left(-\beta \pm \sqrt{\beta^{2}-4 \alpha}\right)\right. \\
& \left.+\frac{1}{4 \alpha^{2}}\left[3 L+Q-b^{-1} E(F+2 K)-3 b \alpha\right]\left(-\beta^{2} \pm \sqrt{\beta^{2}-4 \alpha}\right)^{2}\right) \lambda \\
& +O\left(\lambda^{3 / 2}\right),
\end{aligned}
$$

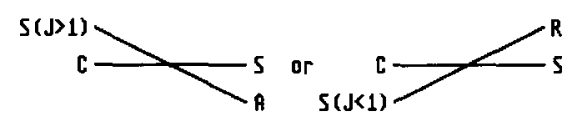

Fig. 12. Schematical representation of the transcritical bifurcations that can occur if $H=O(1)$. An attractor is indicated by (A), a repeller by (R), a saddle by (S) and a center by (C). 


$$
\Phi=\frac{\lambda b}{2 \alpha} \sqrt{\beta^{2}-4 \alpha}\left(\sqrt{\beta^{2}-4 \alpha} \mp \beta\right)+\sigma\left(\lambda^{3 / 2}\right) .
$$

We observe that if $\alpha<0$ the points are at different sides of the origin, one thus having $\mathscr{I}>1$ and the other $\mathscr{I}<1$. Furthermore, both points have $\Phi>0$ if $b<0$ and $\Phi<0$ if $b>0$. The origin itself is a center if $b<0$ and a saddle if $b>0$. So if the origin is a center we have a saddle with $\mathscr{f}<1$ at one side and a saddle with $\mathscr{I}>1$ at the other side of the origin. If, on the other hand, the origin is a saddle (with $\mathscr{I}=1$ ) we have an attractor at one side and a repeller at the other.

If $\alpha>0$ we have both fixed points at the same side of the origin, provided that $\alpha<\beta^{2 / 4}$, otherwise they are not there at all. The points then both have $\mathscr{F}<1$, or $\mathscr{f}>1$. Furthermore, one has $\Phi>0$ and the other has $\Phi<0$. The point closest to the origin has $\Phi<0$ if $b>0$ and vice versa. So if the origin is a center we have, at the same side, a saddle with $f<1$ and an attractor. or a saddle with $\mathscr{f}>1$ and a repeller. The saddle is closest to the origin. If, on the other hand, the origin is a saddle, we have the same possibilities, only now the attractor or repeller is closest to the origin.

For $\lambda$ small and negative one can make analogous considerations. Here we find for the fixed points outside the origin, provided that $\alpha>-\beta^{2 / 4}$.

$$
w=\frac{1}{2 \alpha}\left(-\beta \pm \sqrt{\beta^{2}+4 \alpha}\right) \sqrt{-\lambda}+O(\lambda)
$$

For the Jacobian $\mathscr{I}$ and the Stability $\Phi$ we obtain in terms of $\lambda$ that

$$
\begin{aligned}
\mathscr{I}=1 & +\frac{J+2 E}{2 \alpha}\left(-\beta \pm \sqrt{\beta^{2}+4 \alpha}\right) \sqrt{-\lambda} \\
& +\left(\frac{-b \beta}{\alpha}\left(-\beta \pm \sqrt{\beta^{2}+4 \alpha}\right)\right. \\
& \left.+\frac{1}{4 \alpha^{2}}\left[3 L+Q-b^{1} E(F+2 K)-3 b \alpha\right]\left(-\beta^{2} \pm \sqrt{\beta^{2}+4 \alpha}\right)^{2}\right)(-\lambda) \\
& +\sigma\left(\lambda^{32}\right), \\
\Phi= & \frac{-\lambda b}{2 \alpha} \sqrt{\beta^{2}+4 \alpha}\left(\sqrt{\beta^{2}+4 \alpha} \mp \beta\right)+C\left(\lambda^{3}\right) .
\end{aligned}
$$

We observe that for $\alpha>0$ and $\lambda<0$ we have the same possibilities as for $\alpha<0$ and $\lambda>0$. Also, for $\alpha<0$ and $\lambda<0$ we have the same possibilities as for $\alpha>0$ and $\lambda>0$, only the proviso for the existence is now that $\alpha>-\beta^{2 / 4}$.

Putting this together we find six different bifurcation scenarios, depending on the values of $\alpha$ and $\beta$. In fig. 13 we have listed the various scenarios in a 


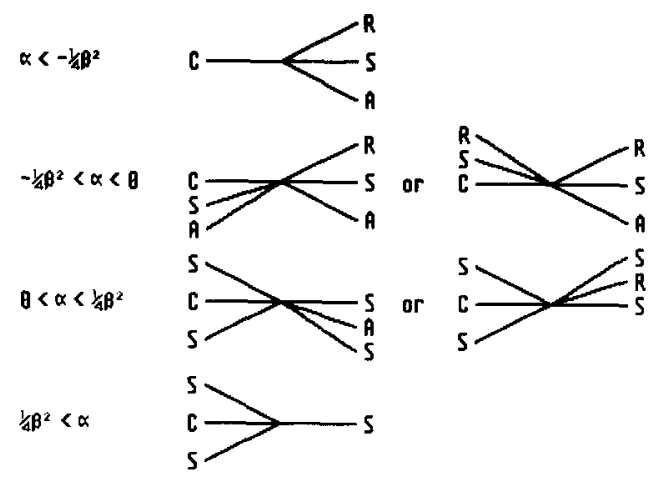

Fig. 13. Schematical representation of the bifurcation scenarios that can occur if $H=\mathscr{O}(\sqrt{|\lambda|})$, for $b>0$ and for various values of $\alpha$ and $\beta$. An attractor is indicated by (A), a repeller by (R), a saddle by $(S)$ and a center by $(C)$. The horizontal lines represent the fixed point at the origin (with $\mathscr{I}=1$ ). The parameter $\lambda$ increases from negative values at the left to positive values at the right. The other lines represent the fixed points outside the origin (with $\mathscr{f} \neq 1$ ). Above the horizontal lines they have $\mathscr{I}>1$, below they have $\mathscr{F}<1$. The order of the fixed points with respect to $w$ is the same as the order with respect to $\mathscr{F}$ (apart from an overall reflection with respect to the horizontal lines).

schematical way for the case $b>0$. The bifurcation scenarios for the case $b<0$ follow from those for $b>0$ by substituting $\alpha \leftrightarrow-\alpha$ and $\lambda \leftrightarrow-\lambda$.

We observe that for $|\alpha|>\beta^{2} / 4$ we have a Rimmer bifurcation.

In the marginal case $\beta^{2}=4|\alpha|$ we have $\Phi=\mathscr{O}\left(\lambda^{3 / 2}\right)$. In order to evaluate $\Phi$ here one needs the $w^{3}$ term in the expansion of $\Phi$, which also contains fourth order coefficients of the mapping. We shall not pursue this point any further here.

\section{B.3.3. $H=\mathscr{O}(\lambda)$}

The fixed points outside the origin exist if

$$
(H-\lambda E)^{2}-4 \alpha \lambda=-4 \alpha \lambda+\mathcal{O}\left(\lambda^{2}\right)>0,
$$

that is, if $\alpha \lambda<0$. We then find for the fixed points

$$
w= \pm \sqrt{\frac{-\lambda}{\alpha}}+\mathcal{O}(\lambda)
$$

For the Jacobian $\mathscr{J}$ and the Stability $\Phi$ we now have in terms of $\lambda$ that

$$
\begin{aligned}
\mathscr{J}= & 1 \pm(J+2 E) \sqrt{\frac{-\lambda}{\alpha}}+\left[3 L+Q-b^{-1} E(F+2 K)+3(E J-b P)\right] \frac{-\lambda}{\alpha} \\
& +\mathcal{O}\left(\lambda^{3 / 2}\right), \\
\Phi= & -2 \lambda b+\mathcal{O}\left(\lambda^{3 / 2}\right) .
\end{aligned}
$$


Thus when $\lambda b$ passes through 0 (from below upward for $\alpha<0$ and from above downward for $\alpha>0$ ) we have that

- If $b>0$ then the origin changes from a center into a saddle (with $f=1$ of course) giving birth to an attractor and a repeller.

- If $b<0$ then the origin changes from a saddle with $\mathscr{F}=1$ into a center giving birth to two saddles, one with $\mathscr{f}>1$ and the other with $\neq<1$.

These are Rimmer types of bifurcations, like the ones schematically depicted in fig. 6.

Finally there is also the possibility that $J+2 E=2 b H=\ell(\lambda)$. Then the fixed points outside the origin will have both $\mathscr{f}>1$ or $\mathscr{f}<1$, according to the sign of $3 L+Q-b{ }^{1} E(F+2 K)+3(E J-b P)$.

\section{References}

[1] K. Kirchgassner, J. Diff. Eqs. 45 (1982) 113.

[2] M. Renardy, Adv. Appl. Math. 3 (1982) 38.4

[3] F.T. Arecchi, Nucl. Phys. B (Proc. Suppl.) 2 (1987) 13.

14] A. Politi, G.L. Oppo and R. Badii. Phys. Rev. A 33 (1986) 4055.

[5] A. Politi. G.L. Oppo and R. Badii. Lecture Notes in Physics, vol. 262 (Springer. Berlin, 1986), p. 486.

[6] G.R.W. Quispel and J.A.G. Roberts. Phys. Lett. A 1.32 (1988) 161.

[7] G.R.W. Quispel and J.A.G. Roberts, Phys. Lett. A 135 (1989) 337.

[8] G.R.W. Quispel, Order and chaos in conservative and in reversible systems. Lectures notes. The Townsville Winter School on Theoretical Physics (1988).

[9] B.V. Chirikov, Phys. Rev. 52 (1979) 265.

[10] J. Guckenheimer and P. Holmes. Nonlinear Oscillations, Dynamical Systems and Bifurcations of Vector Fields (Springer, Berlin, 1983)

[11] R.S. MacKay, Ph.D. Thesis, Princeton University (1982)

[12] R.J. Rimmer, J. Diff. Eqs. 29 (1978) 329.

[13] R.J. Rimmer, Memoirs Am. Math. Soc. 272 (1983) 1.

[14] G.R.W. Quispel and H.W. Capel, Phys. Lett. A 142 (1989) 112.

[15] J. Moser, SIAM Rev. 8 (1966) 145.

[16] J. Moser. Math. Ann. 169 (1967) 136.

[17] M.B. Sevryuk. Reversible Systems, Lecture Notes in Mathematics. vol. 1211 (Springer. Berlin. 1986).

[18] J.A.G. Roberts. Order and chaos in reversible dynamical systems. Ph.D. Thesis, University of Melbourne (1990).

[19] K.M. Pinnow, Reversibele Strömungen. Diplomarbeit, ETH Zürich (1986).

[20] J.P. van der Weele, H.W. Capel, T. Post and Ch.J. Calkoen, Physica A 137 (1986) 1.

[21] G.R.W. Quispel, Phys. Lett. A 112 (1985) 353. 